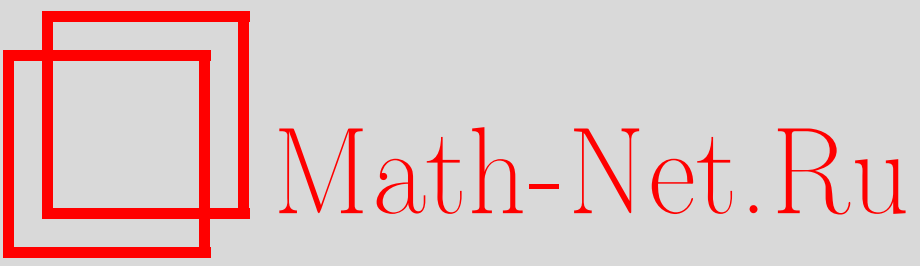

С. Г. Пятков, О некоторых классах обратных задач об определении функции источников для систем тепломассопереноса, Итоги науки и техн. Сер. Соврем. мат. и ее прил. Темат. обз., 2020, том 188, 23-42

DOI: https://doi.org/10.36535/0233-6723-2020-188-23-42

Использование Общероссийского математического портала Math-Net.Ru подразумевает, что вы прочитали и согласны с пользовательским соглашением

http://www.mathnet.ru/rus/agreement

Параметры загрузки:

IP: 18.209 .158 .208

26 апреля 2023 г., $11: 38: 18$ 


\title{
О НЕКОТОРЫХ КЛАССАХ ОБРАТНЫХ ЗАДАЧ ОБ ОПРЕДЕЛЕНИИ ФУНКЦИИ ИСТОЧНИКОВ ДЛЯ СИСТЕМ ТЕПЛОМАССОПЕРЕНОСА
}

\author{
(c) 2020 г \\ С. Г. ПЯтКов
}

\begin{abstract}
АннотАция. В работе рассматривается вопрос о корректности в пространствах Соболева задачи определения функции источников в системе тепломассопереноса, состоящей из системы НавьеСтокса, параболического уравнения для температуры и параболической системы для концентраций переносимых веществ. В качестве условия переопределения берутся интегралы от решения с весом по пространственной области. Доказана локальная (по времени) теорема существования решения задачи в нелинейном случае и получены оценки устойчивости, для линеаризованной системы получена глобальная теорема существования.
\end{abstract}

Ключевые слова: система тепломассопереноса, обратная задача, интегральное переопределение, существование, единственность, начально-краевая задача.

\section{ON SOME CLASSES OF INVERSE PROBLEMS ON DETERMINING SOURCE FUNCTIONS FOR HEAT AND MASS TRANSFER SYSTEMS}

\author{
(c) 2020 S. G. PYATKOV
}

\begin{abstract}
In this paper, we considers the question on the well-posedness in Sobolev spaces of the problem of determining the source function in a heat and mass transfer system consisting of the Navier-Stokes system, a parabolic equation for temperature, and a parabolic system for the concentrations of substances being transferred. Weighted integrals solution over the spatial domain serve as overdetermination conditions. A local (in time) existence theorem for the solution of the problem in the nonlinear case is proved and stability estimates are obtained; for a linearized system, a global existence theorem is obtained.
\end{abstract}

Keywords and phrases: heat and mass transfer system, inverse problem, integral overdetermination, existence, uniqueness, initial-boundary-value problem.

AMS Subject Classification: 35R30, 35K51, 35Q35, 80A20, 80A23

1. Введение. Рассматривается система

$$
\begin{gathered}
u_{t}-\nu \Delta u+(u, \nabla) u+\nabla p=f+\beta_{c} C+\beta_{\theta} \Theta, \quad \operatorname{div} u=0, \\
\Theta_{t}-\lambda_{\theta} \Delta \Theta+(u, \nabla) \Theta=f_{\theta}, \\
C_{t}+(u, \nabla) C-\sum_{i, j=1}^{n} a_{i j} C_{x_{i} x_{j}}+\sum_{i=1}^{n} a_{i} C_{x_{i}}+a_{0} C=f_{c},
\end{gathered}
$$

Работа выполнена при поддержке Российского фонда фундаментальных исследований и правительства ХантыМансийского автономного округа-Югры (проект № 18-41-860003 р_урал_а). 
где $\nu=$ const $>0,(x, t) \in Q=G \times(0, T)\left(G \subset \mathbb{R}^{n}, T<\infty\right), u, \Theta, p, C, f_{c}$ суть вектор скорости, температура жидкости, давление, вектор концентраций примесей (органических или неорганических) в жидкости и объемная плотность источников примесей, соответственно. Здесь $a_{i j}, a_{i}$, $a_{0}$ - матрицы размерности $(h \times h)$, где $h$-это количество примесей, $\beta_{C}$ - матрица размерности $(n \times h), \beta_{\Theta}$ - вектор-функция длины $n, \lambda_{\Theta}>0$ - скалярная функция. Описание таких моделей имеется, например, в [8], где на основе термодинамики необратимых процессов выведена система дифференциальных уравнений тепло- и массопереноса в самом общем виде. В частности, там рассматриваются и системы, где система Навье-Стокса заменена более общей системой. Система (1)-(3) описывает распространение примесей в жидкости. В частности, в класс систем (1)-(3) входит классическая модель Обербека-Буссинеска (см. $[9,20,38,44])$. Функции $f_{\theta}$ и $f$ являются плотностями источников тепла и внешних сил соответственно, а коэффициент $\lambda_{\theta}$ есть коэффициент теплопроводности. В модели Обербека-Буссинеска $\beta_{c}$ и $\beta_{\theta}$ суть коэффициенты переноса массы и тепла, умноженные на ускорение свободного падения. Здесь считаем, что $\beta_{c}$ - произвольная матрица-функция размерности $(n \times h)$ и $\beta_{\theta}$ - вектор-функция длины $n$.

Система (1)-(3) дополняется начальными и граничными условиями

$$
\begin{gathered}
\left.u\right|_{t=0}=u_{0},\left.\quad u\right|_{S}=0, \quad \Gamma=\partial G, \quad S=\Gamma \times(0, T), \\
\left.\Theta\right|_{t=0}=\Theta_{0},\left.\quad B_{1} \Theta\right|_{S}=g_{2}(t, x),\left.\quad C\right|_{t=0}=C_{0},\left.\quad B_{2} C\right|_{S}=g_{3}(t, x),
\end{gathered}
$$

где

$$
B_{1} u=u \quad \text { или } \quad B_{1} u=\sum_{i=1}^{n} \gamma_{1 i}(x, t) \frac{\partial u}{\partial x_{i}}+\sigma_{1}(x, t) u,
$$

$\gamma_{1 i}, \sigma_{1}$ - некоторые функции,

$$
B_{2} u=u \quad \text { или } \quad B_{2} u=\sum_{i=1}^{n} \gamma_{2 i}(x, t) \frac{\partial u}{\partial x_{i}}+\sigma_{2}(x, t) u,
$$

$\gamma_{2 i}, \sigma_{2}$ - некоторые матрицы-функции размерности $(h \times h)$ и $S=(0, T) \times \Gamma$. Таким образом, мы рассматриваем одно из классических граничных условий Дирихле, Неймана, Робена или условие с косой производной. Правая часть в (3) имеет вид

$$
f_{c}=f_{0}(x, t)+\sum_{i=1}^{s} f_{i}(x, t) q_{i}(t), \quad(x, t) \in Q,
$$

где $f_{i}(i=0,1, \ldots, m)$ - известные функции. Числовые функции $q_{i}(t)$ в данном представлении неизвестны и находятся с использованием условий переопределения:

$$
\int_{G}\left(C, \varphi_{i}(x)\right) d x=\psi_{i}(t), \quad i=1,2, \ldots, s,
$$

где скобки обозначают скалярное произведение в $\mathbb{R}^{h}, \varphi_{i}(x)$ - вектор-функции длины $h, \psi_{i}(t)$ некоторые функции.

Обратные задачи такого типа возникают в химии, биологии и других областях при описании процессов тепломассопереноса, диффузионных процессов, процессов фильтрации. Описание некоторых численных методов решения краевых задач для системы (1)-(3) можно найти в [9]. Отметим работу [1], где для системы (1)-(3) рассмотрено большое количество обратных и экстремальных задач в стационарном случае. Подобные модели, в упрощённой постановке, рассматривались в [23-48]. Отметим, что в реальных моделях, используемых в региональных системах поддержки принятия решений, даже в одномерном случае используется несколько уравнений относительно различных примесей в жидкости. В них учитываются такие параметры воды, как фитопланктон, эпифитон и различные химические составляющие (различные подходы к построению таких моделей и библиография описаны в [24]). Вопросы корректности обратных задач для параболических уравнений и систем с условиями переопределения вида (7) (включая численные методы) рассматривались во многих работах. По-видимому, одной из первых работ где 
рассматривался вопрос об определении правой части вида $f=q(t) g(x, t)+f_{0}(x, t)$ в параболическом уравнении (неизвестной функцией является функция $q(t)$ ) была работа [10]. В качестве основных пространств рассматривались пространства Гельдера. Далее, вопрос об определении правой части такого вида по интегральным условиям рассматривался в $[4,22,28,35,37]$. Вопросы определения того или иного коэффициента, зависящего от времени, в параболическом уравнении по интегральным условиям переопределения был рассмотрен, например, в $[3,5,40,41,43,46]$ и многих других. Выделим книгу [42], где в случае $n=1$, был исследован ряд коэффициентных обратных задач (см., например, [42, теорема 4.114]). Стоит отметить, что работ, посвященных многомерным обратных задачам в более или менее общих постановках, мало. Можно отметить книгу [50], где рассматривались задачи такого типа и имеется обширная библиография. Опишем ряд результатов из этой книги. Была рассмотрена задача с условиями переопределения вида (7) в задаче определения функции источника вида $f(t) g(x, t)$ в модельном параболическом уравнении второго порядка (см. $[50, \S 1.5])$. Та же задача была рассмотрена отдельно для системы НавьеСтокса (см. $[50, \S 4.4])$ и ее аналог для операторно-диференциального уравнения первого порядка вида

$$
u_{t}-A(t) u=\Phi(t) p(t)+F(t),
$$

где $A(t)$ - оператор, вообще говоря, с постоянной областью определения, являющийся генератором аналитической полугруппы или полугруппы класса $C_{0}$ (см. $\left.[50, \S 6.2,6.6]\right)$. Был рассмотрен и случай, когда неизвестная функция $p(t)$ входит в правую часть нелинейно а сама задача является квазилинейной. Задача в аналогичной постановке была рассмотрена и в работе автора [13], в случае когда область определения $A(t)$ может зависеть от времени и условия гладкости на этот оператор минимальны. Среди последних работ выделим результаты работ [11-51], где задачи вида (1)-(7) рассмотрены для параболических систем уравнений, как в случае определения функции источника, так и в случае коэффициентных обратных задач. Среди монографий, посвященных обратным задачам для параболических и эллиптических уравнений и систем, можно отметить монографии $[2,21,39,47,49,50]$, в которых можно найти постановки и ряд результатов. Обратные задачи для системы (1)-(3) с данными переопределения на пространственных многообразиях или в отдельных точках были рассмотрены в $[6,52]$. Данная работа является дополнением к уже полученным в указанных работах результатах. Мы приведем теоремы существования и единственности и оценки устойчивости решений, как в нелинейной, так и в линеаризованной постановке.

2. Обозначения и вспомогательные утверждения. Пусть $E$-банахово пространство. Обозначим через $L_{p}(G ; E)$ ( $G$ область $\left.\mathbb{R}^{n}\right)$ пространство сильно измеримых функций, определенных на $G$ со значениями в $E$ и конечной нормой \|\|$u(x)\left\|_{E}\right\|_{L_{p}(G)}$ (см., например, [54, §1.18.4]). Также используются пространства $C^{k}(\bar{G} ; E)$ состоящие из функций, обладающих всеми производными до $k$ включительно, непрерывных и ограниченных в $G$, имеющих непрерывное продолжение на $\bar{G}$. Пространства Соболева $W_{p}^{k}(G ; E), W_{p}^{k}(Q ; E)$ определены стандартным образом (см. $[18,19,25,54])$. Если $E=\mathbb{C}$ или $E=\mathbb{C}^{n}$, то используется обозначение $W_{p}^{k}(G)$, или $C^{k}(\bar{G})$. Принадлежность $u \in W_{p}^{k}(G)$ (или $u \in C^{k}(\bar{G})$ ) для заданной вектор-функции $u=\left(u_{1}, u_{2}, \ldots, u_{k}\right)$ означает, что каждая компонента $u_{i}$ принадлежит $W_{p}^{k}(G)$ (или $C^{k}(\bar{G})$ ). Норма в соответствующем пространстве - сумма норм координат, если не указано противное. Аналогичное соглашение примем для матриц: принадлежность $a \in W_{p}^{k}(G)\left(a=\left\{a_{i j}\right\}_{j, i=1}^{k}\right)$ означает, что $a_{i j}(x) \in W_{p}^{k}(G)$ для всех $i, j$. Для заданного интервала $J=(0, T)$ положим

$$
W_{p}^{k, r}(Q)=W_{p}^{r}\left(J ; L_{p}(G)\right) \cap L_{p}\left(J ; W_{p}^{k}(G)\right), \quad W_{p}^{k, r}(S)=W_{p}^{r}\left(J ; L_{p}(\Gamma)\right) \cap L_{p}\left(J ; W_{p}^{k}(\Gamma)\right) .
$$

Обозначим через $L_{p, \sigma}(G)$ замыкание соленоидальных $C_{0}^{\infty}$-вектор-функций по норме $L_{p}(G)$ и положим

$$
W_{p, \sigma}^{k}(G)=W_{p}^{k}(G) \cap L_{p, \sigma}(G), \quad W_{p, \sigma}^{k, k / 2}(Q)=W_{p}^{k, k / 2}(Q) \cap L_{p}\left(0, T ; L_{p, \sigma}(G)\right), \quad k \geqslant 0 .
$$


Символ $\stackrel{\circ}{W_{q}^{k}}(G)$ обозначает замыкание $C_{0}^{\infty}(G)$ по норме пространства $W_{q}^{k}(G)$ и

$$
\dot{W}_{q}^{1}(G)=\left\{p \in L_{q, l o c}(G): \nabla p \in L_{q}(G)\right\} .
$$

Мы отождествляем функции, отличающиеся на константу, и вводим в этом пространстве норму $\|p\|_{\dot{W}_{q}^{1}(G)}=\|\nabla p\|_{L_{q}(G)}$; это пространство является банаховым. Символы $(A, B)_{\theta, q}$ и $[A, B]_{\theta}$ для заданных банаховых пространств $A, B$ обозначают пространства, полученные при помощи вещественного и комплексного интерполяционных методов (см. [54]). Символ $\partial_{x_{k}}$ обозначает частную производную $\partial / \partial x_{k}$.

Определение границы класса $C^{s}$ стандартное (см., например, [7, гл. $1, \S 1$, п. 3]). В дальнейшем используем обозначения $Q^{\tau}=G \times(0, \tau), S^{\tau}=\Gamma \times(0, \tau)$.

Лемма 1. Пусть $u \in W_{q}^{2,1}\left(Q^{\tau}\right), q \in(1, \infty), u u(x, 0)=0$. Тогда существует такая константа $c>0$, не зависящая от $и$, что

$$
\|u\|_{L_{q}\left(Q^{\tau}\right)} \leqslant c \tau\|u\|_{W_{q}^{2,1}\left(Q^{\tau}\right)}, \quad\|\nabla u\|_{L_{q}\left(Q^{\tau}\right)} \leqslant c \tau^{1 / 2}\|u\|_{W_{q}^{2,1}\left(Q^{\tau}\right)} .
$$

Утверждение является следствием формулы Ньютона-Лейбница и интерполяционного неравенства

$$
\|\nabla u\|_{L_{q}(G)} \leqslant c\|u\|_{W_{q}^{2}(G)}^{1 / 2}\|u\|_{L_{q}(G)}^{1 / 2} .
$$

Следующая лемма является следствием леммы 1 и [7, гл. 2, лемма 3.3], где $\delta=\sqrt{\tau}$.

Лемма 2. Пусть $u \in W_{q}^{2,1}\left(Q^{\tau}\right)$. Тогда

$$
\begin{aligned}
& u \in L_{p}\left(Q^{\tau}\right), \quad \text { әде } \quad 2-l\left(\frac{1}{q}-\frac{1}{p}\right)(n+2) \geqslant 0, \quad p \geqslant q, \\
& \nabla u \in L_{p}\left(Q^{\tau}\right), \quad \text { где } 1-l\left(\frac{1}{q}-\frac{1}{p}\right)(n+2) \geqslant 0, \quad p \geqslant q .
\end{aligned}
$$

Более того,

$$
\begin{array}{lll}
u \in C^{\lambda, \lambda / 2}\left(\overline{Q^{\tau}}\right) & \partial л \Omega & q>\text { fracn }+22, \\
\nabla u \in C^{\lambda, \lambda / 2}\left(\overline{Q^{\tau}}\right) & \partial л я & q>n+2,
\end{array}
$$

где $\lambda \in[0,2-(n+2) / q)$ в первом случае и $\lambda \in[0,1-(n+2) / q)$ в последнем. Справедливъ следующие оченки для соответствующих значений параметров:

$$
\begin{aligned}
\|u\|_{L_{p}\left(Q^{\tau}\right)} & \leqslant c \tau^{\beta_{1}}\|u\|_{W_{q}^{2,1}\left(Q^{\tau}\right)}, \quad\|\nabla u\|_{L_{p}\left(Q^{\tau}\right)} \leqslant c \tau^{\beta_{1}-1 / 2}\|u\|_{W_{q}^{2,1}\left(Q^{\tau}\right)}, \\
\|u\|_{C^{\lambda, \lambda / 2}\left(\overline{Q^{\tau}}\right)} & \leqslant c \tau^{\beta_{2}}\|u\|_{W_{q}^{2,1}\left(Q^{\tau}\right)}, \quad\|\nabla u\|_{C^{\lambda, \lambda / 2}\left(\overline{Q^{\tau}}\right)} \leqslant c \tau^{\beta_{2}-1 / 2}\|u\|_{W_{q}^{2,1}\left(Q^{\tau}\right)},
\end{aligned}
$$

где

$$
\beta_{1}=1-\frac{(n+2)}{2}\left(\frac{1}{q}-\frac{1}{p}\right), \quad \beta_{2}=1-\frac{(n+2)}{2 q}-\frac{\lambda}{2},
$$

константа с не зависит от $\tau \leqslant T u u \in W_{q}^{2,1}\left(Q^{\tau}\right)$.

Лемма 3. Пусть $b \in L_{p}(Q)$. Тогда для любого $\tau \in(0, T]$ имеют место следующие неравенства: если $q>(n+2) / 2$ u $p \geqslant q$, то

$$
\|b u\|_{L_{q}\left(Q^{\tau}\right)} \leqslant c \tau^{1-(n+2) /(2 p)}\|u\|_{W_{q}^{2,1}\left(Q^{\tau}\right)}
$$

если $q>n+2 u p \geqslant q$, mo

$$
\|b \nabla u\|_{L_{q}\left(Q^{\tau}\right)} \leqslant c \tau^{1 / 2-(n+2) /(2 p)}\|u\|_{W_{q}^{2,1}\left(Q^{\tau}\right)} .
$$

Константа с $>0$ не зависит от $\tau \leqslant T$ и $u \in W_{q}^{2,1}\left(Q^{\tau}\right)$.

Доказательство данной леммы можно найти в доказательстве теоремы 9.1 в [7, гл. 4]. 
Теорема 1. Для каждой функиии $f \in L_{r}(Q), r \in(1, \infty$, существуют единственная векторфункиия $и \in W_{r, \sigma}^{2,1}(Q) \cap L_{r}\left(0, T ; \stackrel{\circ}{W}{ }_{r}^{1}(G)\right)$ и функиия $p \in L_{r}\left(0, \tau ; \dot{W}_{r}^{1}(G)\right)$, являющиеся решением задачи

$$
u_{t}-\nu \Delta u+\nabla p=f, \quad \operatorname{div} u=0,\left.\quad u\right|_{S}=0,\left.\quad u b\right|_{t=0}=0,
$$

и удовлетворяющие неравенству

$$
\|u\|_{W_{r}^{2,1}(Q)}+\|\nabla p\|_{L_{r}(Q)} \leqslant c\|f\|_{L_{r}(Q)}
$$

где постоянная с не зависит от $f$.

Следствие 1. Для каждой функиии $f \in L_{r}\left(Q^{\tau}\right), \tau \in(0, T]$, существуют единственная вектор-функиия $u \in W_{r, \sigma}^{2,1}\left(Q^{\tau}\right) \cap L_{r}(0, \tau ; \stackrel{\circ}{W} \underset{r}{1}(G))$ и функиия $p \in L_{r}\left(0, \tau ; \dot{W}_{r}^{1}(G)\right)$, являюшиеся решением задачи

$$
u_{t}-\nu \Delta u+\nabla p=f, \quad \operatorname{div} u=0,\left.\quad u\right|_{S}=0,\left.\quad u\right|_{t=0}=0,
$$

и удовлетворяющие неравенству

$$
\|u\|_{W_{r}^{2,1}\left(Q^{\tau}\right)}+\|\nabla p\|_{L_{r}\left(Q^{\tau}\right)} \leqslant c\|f\|_{L_{r}\left(Q^{\tau}\right)}
$$

где постоянная с не зависит от $f u \tau$.

Теорема 1 вытекает из [53, теорема 1.1]; также можно сослаться на [31, теорема 1.2] и свойства проектора Гельмгольца.

В случае, когда $G$-ограниченная область, теорема 1 справедлива для любого $r$. При определенных условиях на $r$ теорему можно установить и для более широкого класса областей, в частности, для бесконечного цилиндра (см. [29]), для полупространств (см. [26]), для целого пространства и ограниченных областей с границей класса $C^{1,1}$ (см. [31]), для плоского слоя и асимптотически плоского слоя (см. $[15,16])$ и для некоторых других классов неограниченных областей (см. $[17,32,33])$. Теорема 1 является основной теоремой, используемой ниже.

Следующий результат - теорема о разрешимости параболических задач. Рассмотрим задачу

$$
u_{t}-L u=f,\left.\quad B u\right|_{S}=u_{0}, \quad u(x, 0)=g(x, t),
$$

где

$$
L u=\sum_{i, j=1}^{n} a_{i j}(x, t) u_{x_{i} x_{j}}-\sum_{i=1}^{n} a_{i}(x, t) u_{x_{i}}-a_{0}(x, t) u
$$

$B u=u$ или

$$
B u=\sum_{i=1}^{n} \gamma_{i}(x, t) \frac{\partial u}{\partial x_{i}}+\sigma(x, t) u,
$$

где $\gamma_{i}, \sigma$ - некоторые матрицы-функции размерности $h \times h$ и $S=(0, T) \times \Gamma ; a_{i j}, a_{i}, a_{0}$ - матрицы размера $h \times h$. Предположим, что выполнены условия параболичности и Лопатинского.

Условие параболичности. Пусть

$$
A_{0}(t, x, \xi)=\sum_{i, j=1}^{n} a_{i j}(t, x) \xi_{i} \xi_{j}, \quad \xi \in \mathbb{R}^{n} .
$$

Предположим, что найдется такая постоянная $\delta_{1}>0$, что все корни $p$ полинома $\operatorname{det}\left(A_{0}(t, x, i \xi)+\right.$ $p E)=0$ (здесь $E$ - единичная матрица) удовлетворяют условию

$$
\operatorname{Re} p \leqslant-\delta_{1}|\xi|^{2}
$$

для всех $\xi \in \mathbb{R}^{n}$ и всех $(x, t) \in Q$.

Пусть $B_{0} u=u$ в случае условий Дирихле в $(2)$ и $B_{0} u=\sum_{j=1}^{n} \gamma_{j} \partial_{x_{j}} u$ в противном случае. 
Условие Лопатинского (см. условие (LS) в [27, с. 198]). Для любой точки $\left(t_{0}, x_{0}\right) \in S$, таких векторов $\xi \in \mathbb{R}^{n}$, что $\left(\xi, \nu\left(x_{0}\right)\right)=0$ (здесь $\nu(x)$ - внешняя единичная нормаль к $\Gamma$ в точке $x$ ), всех $\boldsymbol{h} \in \mathbb{C}^{h}$ и таких $\lambda$, что $\operatorname{Re} \lambda \geqslant 0$ и $|\xi|+|\lambda| \neq 0$, система обыкновенных дифференциальных уравнений

$$
\left(\lambda E+A_{0}\left(x_{0}, t_{0}, \xi+i \nu\left(x_{0}\right) \partial_{y}\right)\right) v(z)=0, \quad B_{0}\left(x_{0}, t_{0}, \xi+i \nu\left(x_{0}\right) \partial_{y}\right) v(0)=h
$$

имеет единственное убывающее на бесконечности решение класса $C([0, \infty))$.

Считаем, что

$$
\begin{gathered}
a_{i j} \in C(\bar{Q}), \quad a_{i} \in L_{q}(Q), \quad a_{0} \in L_{q}(Q), \quad i, j=1 \ldots n, \\
\gamma_{j}, \sigma \in C^{1 / 2-1 / 2 p+\varepsilon_{0}, 1-1 / p+\varepsilon_{0}}(\bar{S}), \quad j=1, \ldots, n,
\end{gathered}
$$

для некоторого $\varepsilon_{0}>0$,

$$
u_{0}(x) \in W_{p}^{2-2 / p}(G), \quad g(x, t) \in W_{p}^{s_{0}, 2 s_{0}}(S),\left.\quad B(x, 0, D) u_{0}\right|_{\Gamma}=g(x, 0),
$$

где $s_{0}=1-1 / 2 p$ в случае задачи Дирихле и $s_{0}=1 / 2-1 / 2 p$ в случае оставшихся краевых условий.

Теорема 2. Пусть $\partial G \in C^{2}, q>n+2, \gamma \in(0, T]$ и условия параболичности и Лопатинского, а также (12), (13) выполнены. Тогда для каждого $f \in L_{q}(Q)$ существует единственное решение $u \in W_{q}^{2,1}\left(Q^{\gamma}\right)$ задачи (9), удовлетворяющее оценке

$$
\|u\|_{W_{p}^{1,2}(Q)} \leqslant c\left(\|f\|_{L_{p}(Q)}+\|g\|_{W_{p}^{s_{0}, 2 s_{0}}(S)}+\mid u_{0} \|_{W_{p}^{2-2 / p}(G)}\right)
$$

где постоянная с не зависит от $f, u_{0}, g$.

Теорема является следствием [7, гл. 7, теорема 10.4] (см. также [14] или [27, теорема 2.1]).

Теорема 3. Пусть $\partial G \in C^{2}, q>n+2, u_{0}=0, g=0, \gamma \in(0, T]$ и условия параболичности, Лопатинского и (12) выполнены. Тогда для каждого $f \in L_{q}(Q)$ существует единственное решение $и \in W_{q}^{2,1}\left(Q^{\gamma}\right)$ задачи (9), удовлетворяющее оценке

$$
\|u\|_{W_{q}^{2,1}\left(Q^{\gamma}\right)} \leqslant c\|f\|_{L_{q}\left(Q^{\gamma}\right)}
$$

где константа с не зависит от $\gamma \in(0, T)$.

Данная теорема - очевидное следствие теоремы 2. Мы применяем теорему 2, где берем нулевые начальные и граничные данные и правую часть $f_{\gamma}=f(x, t)$ при $t \in(0, \gamma)$ и $f_{\gamma}=0$ при $t \in(\gamma, T)$.

3. Формулировка основных результатов. Всюду ниже считаем, что $q>n+2$.

Условие согласования и гладкости могут быть записаны в следующей форме:

$$
\begin{gathered}
u_{0} \in W_{q}^{2-2 / q}(G), \quad \operatorname{div} u_{0}=0,\left.\quad u_{0}\right|_{\Gamma}=0 ; \\
C_{0}(x) \in W_{q}^{2-2 / q}(G), \quad g_{3}(x, t) \in W_{q}^{s_{0}, 2 s_{0}}(S),\left.\quad B_{2}(x, 0, D) C_{0}\right|_{\Gamma}=g_{3}(x, 0) ; \\
\Theta_{0}(x) \in W_{q}^{2-2 / q}(G), \quad g_{2}(x, t) \in W_{q}^{1-1 / 2 q, 2-1 / q}(S),\left.\quad B_{1}(x, 0, D) \Theta_{0}\right|_{\Gamma}=g_{2}(x, 0) ; \\
f, f_{\theta}, f_{0} \in L_{q}(Q) ; \\
\psi_{i}(t) \in W_{q}^{1}(0, T), \quad \psi_{i}(0)=\int_{G_{i}} C_{0}(x) \varphi_{i}(x) d x, \quad i=1,2, \ldots, s ; \\
a_{i}(t, x) \in L_{q}(Q), i=0,1, \ldots, n ; \quad a_{i j} \in C\left([0, T] ; C^{\varepsilon_{0}}(\bar{G}), \quad i, j=1, \ldots, n,\right. \\
\gamma_{i j}, \sigma \in C^{1 / 2-1 / 2 p+\varepsilon_{0}, 1-1 / p+\varepsilon_{0}}(\bar{S}), \quad j=1, \ldots, n, i=1,2,
\end{gathered}
$$

где $\varepsilon_{0}>0$ - некоторая положительная постоянная;

$$
f_{i}(x, t) \in L_{\infty}\left(0, T ; L_{q}(G)\right), \quad i=1,2, \ldots, s .
$$

Пусть $\left\{G_{j}\right\}$ - набор областей с границей класса $C^{1}$, вложенных в $G$. Предположим, что

$$
\varphi_{j} \in W_{r}^{\varepsilon_{1}}(G), \quad \frac{1}{q}+\frac{1}{r}=1, \quad j=1,2, \ldots, s,
$$

для некоторого $\varepsilon_{1}>0$. 
Определим элементы $b_{i j}(t)$ матрицы $B$ равенством

$$
b_{i j}=\int_{G}\left(f_{j}, \varphi_{i}(x)\right) d x .
$$

Полагаем, что существует такая постоянная $\delta_{0}>0$, что

$$
|\operatorname{det} B| \geqslant \delta_{0}>0 \quad \text { п.в. на }(0, T) .
$$

Также будем считать, что найдется такая постоянная $\delta_{1}>0$, что

$$
\left.\lambda_{\theta}(x, t) \geqslant \delta_{1}>0, \quad(x, t) \in Q\right), \quad \lambda_{\theta} \in C(\bar{Q}) ; \quad \beta_{c}, \beta_{\theta} \in L_{q}(Q) .
$$

Введем множество $B_{R}$ данных $\boldsymbol{U}=\left(u_{0}, C_{0}, \Theta_{0}, g_{2}, g_{3}, f, f_{0}, f_{\theta}, \psi_{1}, \ldots, \psi_{s}\right)$, удовлетворяющих условиям (14)-(18) и неравенству

$$
\begin{aligned}
\left\|u_{0}\right\|_{W_{q}^{2-2 / q}(G)}+\left\|C_{0}\right\|_{W_{q}^{2-2 / q}(G)}+\left\|\Theta_{0}\right\|_{W_{q}^{2-2 / q}(G)}+\left\|g_{2}\right\|_{W_{q}^{1-1 / 2 q, 2-1 / q}(S)}+ \\
\quad+\left\|g_{3}\right\|_{W_{q}^{s_{0}, 2 s_{0}}(S)}+\|f\|_{L_{q}(Q)}+\left\|f_{\theta}\right\|_{L_{q}(Q)}+\left\|f_{0}\right\|_{L_{q}(Q)}+\sum_{i=1}^{s}\left\|\psi_{i}\right\|_{W_{q}^{1}(0, T)} \leqslant R
\end{aligned}
$$

где функция $\Phi_{1}$ удовлетворяет условиям (14).

Теорема 4. Пусть $\Gamma \in C^{2}, p>n+2$, и выполнены условия (19)-(24). Фиксируем $R_{0}>0$. Тогда существует такое число $\tau_{0}=\tau_{0}\left(R_{0}\right) \in(0, T]$, что для каждого набора данных $\boldsymbol{U}$ из множества $B_{R_{0}}$ существует единственное решение $\left(u, p, \Theta, C, q_{1}, \ldots, q_{m}\right)$ задачи (1)-(7) из класса

$$
u \in W_{q}^{2,1}\left(Q^{\tau_{0}}\right), \quad p \in L_{q}\left(0, \tau_{0} ; \dot{W}_{q}^{1}(G)\right), \quad q_{j} \in L_{q}\left(Q_{0}^{\tau_{0}}\right), \quad j=1,2, \ldots, s,
$$

u найдется такая постоянная $c=c\left(R_{0}\right)$, что для любых двух решений $u^{i}, \Theta^{i}, C^{i}, q^{i}, q^{i}=$ $\left(q_{i 1}, q_{i 2}, \ldots, q_{i m}\right), i=1,2$, из этого класса, отвечающих наборам

$$
\boldsymbol{U}_{1}, \boldsymbol{U}_{2} \in B_{R_{0}}, \quad \boldsymbol{U}_{i}\left(u_{0}^{i}, C_{0}^{i}, \Theta_{0}^{i}, g_{2}^{i}, g_{3}^{i}, f^{i}, f_{0}^{i}, f_{\theta}^{i}, \psi_{1}^{i}, \ldots, \psi_{s}^{i}\right), \quad i=1,2,
$$

справедлива оченка

$$
\begin{gathered}
\left\|u^{1}-u^{2}\right\|_{W_{q}^{2,1}\left(Q^{\tau_{0}}\right)}+\left\|\Theta^{1}-\Theta^{2}\right\|_{W_{q}^{2,1}\left(Q^{\tau_{0}}\right)}+\left\|C^{1}-C^{2}\right\|_{W_{q}^{2,1}\left(Q^{\tau_{0}}\right)}+\sum_{j=1}^{s}\left\|q_{1 j}-q_{2 j}\right\|_{L_{q}\left(0, \tau_{0}\right)} \leqslant \\
\leqslant c\left(\left\|u_{0}^{1}-u_{0}^{2}\right\|_{W_{q}^{2-2 / q}(G)}+\left\|f^{1}-f^{2}\right\|_{L_{q}\left(Q^{\tau_{0}}\right)}+\left\|f_{\theta}^{1}-f_{\theta}^{2}\right\|_{L_{q}\left(Q^{\tau_{0}}\right)}+\left\|f_{0}^{1}-f_{0}^{2}\right\|_{L_{q}\left(Q^{\tau_{0}}\right)}+\right. \\
\quad+\left\|C_{0}^{1}-C_{0}^{2}\right\|_{W_{q}^{2-2 / q}(G)}+\left\|\Theta_{0}^{1}-\Theta_{0}^{2}\right\|_{W_{q}^{2-2 / q}(G)}+\left\|g_{2}^{1}-g_{2}^{2}\right\|_{W_{q}^{1-1 / 2 q, 2-1 / q}\left(S^{\tau_{0}}\right)}+ \\
\left.+\left\|g_{3}^{1}-g_{3}^{2}\right\|_{W_{q}^{s_{0}, 2 s_{0}}\left(S^{\tau_{0}}\right)}+\sum_{i=1}^{s}\left\|\psi_{i}^{1}-\psi_{i}^{2}\right\|_{W_{q}^{1}\left(0, \tau_{0}\right)}\right) .
\end{gathered}
$$

Рассмотрим линеаризованную постановку. Рассматривается система уравнений

$$
\begin{gathered}
u_{t}-\nu \Delta u+\nabla p=\sum_{j=1}^{n} B_{j} u_{x_{j}}+B_{0} u+f+\beta_{C} C+\beta_{\theta} \Theta, \quad \operatorname{div} u=0, \\
\Theta_{t}-\lambda_{\theta} \Delta \Theta+\sum_{j=1}^{n} b_{j} \Theta_{x_{j}}+b_{0} \Theta=f_{\theta}+\sum_{j=1}^{n} b^{j} u_{j}, \\
C_{t}-\sum_{i, j=1}^{n} a_{i j} C_{x_{i} x_{j}}+\sum_{j=1}^{n} a_{j} C_{x_{j}}+a_{0} C=f_{c}+\sum_{j=1}^{n} a^{j} u_{j} .
\end{gathered}
$$

Полагаем далее, что

$$
B_{0}, b^{j}, b_{0}, a^{j}, B_{j}, b_{j}, \in L_{q}(Q), \quad j=1,2, \ldots, n .
$$


Теорема 5. Пусть $\Gamma \in C^{2}, p>n+2$, выполнены условия (19)-(24), (28) и набор данных $\boldsymbol{U}$ удовлетворяет условиям (14)-(18). Тогда существует единственное решение $\left(u, p, \Theta, C, q_{1}, \ldots, q_{m}\right)$ задачи (25)-(27), (4)-(7) из класса

$$
u \in W_{q}^{2,1}(Q), \quad p \in L_{q}\left(0, T ; \dot{W}_{q}^{1}(G)\right), \quad q_{j} \in L_{q}(Q), \quad j=1,2, \ldots, s .
$$

Решение удовлетворяет ощенке

$$
\begin{gathered}
\|u\|_{W_{q}^{2,1}(Q)}+\|\Theta\|_{W_{q}^{2,1}(Q)}+\|C\|_{W_{q}^{2,1}(Q)}+\sum_{j=1}^{s}\left\|q_{j}\right\|_{L_{q}(0, T)} \leqslant \\
\leqslant c\left(\left\|u_{0}\right\|_{W_{q}^{2-2 / q}(G)}+\|f\|_{L_{q}(Q)}+\left\|f_{\theta}\right\|_{L_{q}(Q)}+\left\|f_{0}\right\|_{L_{q}(Q)}+\left\|C_{0}\right\|_{W_{q}^{2-2 / q}(G)}+\left\|\Theta_{0}\right\|_{W_{q}^{2-2 / q}(G)}+\right. \\
\left.\left\|g_{2}\right\|_{W_{q}^{1-1 / 2 q, 2-1 / q}(S)}+\left\|g_{3}\right\|_{W_{q}^{s_{0}, 2 s_{0}}(S)}+\sum_{i=1}^{s}\left\|\psi_{i}^{1}-\psi_{i}^{2}\right\|_{W_{q}^{1}(0, T)}\right) .
\end{gathered}
$$

4. Доказательство основных результатов. Доказательство теоремъ 4. Построим функции $\Phi_{1}, \Phi_{2}, \Phi_{3} \in W_{q}^{2,1}(Q)$, удовлетворяющие начально-краевым условиям (4), (5), т.е.

$$
\left.\Phi_{1}\right|_{t=0}=u_{0},\left.\quad \Phi_{2}\right|_{t=0}=\Theta_{0},\left.\quad \Phi_{3}\right|_{t=0}=C_{0},\left.\quad \Phi_{1}\right|_{S}=0,\left.\quad \Phi_{i}\right|_{S}=g_{i}, \quad i=2,3 .
$$

Используя теоремы 1,2 , строим эти функции как решения соответствующих начально-краевых задач с нулевыми правыми частями. Сделаем замену $u=v+\Phi_{1}, \Theta=\Theta_{1}+\Phi_{2}$, и $C=C_{1}+\Phi_{3}$. Получим

$$
\begin{gathered}
L_{01}(v, p)=v_{t}-\nu \Delta v+\nabla p=g+\beta_{c} C_{1}+\beta_{\theta} \Theta_{1}-\left(\Phi_{1}, \nabla\right) v-(v, \nabla) v-(v, \nabla) \Phi_{1}, \\
\quad \operatorname{div} v=0 \\
L_{02} \Theta_{1}=\Theta_{1 t}-\lambda_{\theta} \Delta \Theta_{1}=g_{\theta}-(v, \nabla) \Theta_{1}-\left(\Phi_{1}, \nabla\right) \Theta_{1}-(v, \nabla) \Phi_{2}, \\
L_{03} C_{1}=C_{1 t}-\sum_{i, j=1}^{n} a_{i j} C_{1 x_{i} x_{j}}+\sum_{j=1}^{n} a_{j} C_{1 x_{j}}+a_{0} C_{1}= \\
=g_{c}-(v, \nabla) C_{1}-\left(\Phi_{1}, \nabla\right) C_{1}-(v, \nabla) \Phi_{3}+\sum_{j=1}^{m} f_{j} q_{j},
\end{gathered}
$$

где новая функция $g_{\theta}$ и вектор-функции $g, g_{c}$ имеют вид

$$
\begin{gathered}
g=f-\Phi_{1 t}+\nu \Delta \Phi_{1}-\left(\Phi_{1}, \nabla\right) \Phi_{1}+\beta_{c} \Phi_{3}+\beta_{\theta} \Phi_{2}, \\
g_{\theta}=f_{\theta}-L_{02} \Phi_{2}-\left(\Phi_{1}, \nabla\right) \Phi_{2}, \quad g_{c}=f_{0}-L_{03} \Phi_{3}-\left(\Phi_{1}, \nabla\right) \Phi_{3} .
\end{gathered}
$$

Новые функции $v, \theta_{1}$ и вектор-функция $C_{1}$ удовлетворяют однородным краевым условиям (4), (5), (7), причем выполнены условия переопределения

$$
\int_{G}\left(C_{1}, \varphi_{j}(x)\right) d x=\psi_{j}(t)-\int_{G}\left(\Phi_{3}, \varphi_{j}(x)\right) d x=\tilde{\psi}_{j}(t), \quad i=1,2, \ldots, s .
$$

Мы свели задачу к эквивалентной задаче. Пусть $\gamma \in(0, T]$. Используя теоремы 1 , 2, можем переписать уравнения (29), (30), (31) в следующей форме:

$$
\begin{gathered}
(v, p)=\left(L_{01}\right)^{-1} g+\left(L_{01}\right)^{-1}\left(\beta_{c} C_{1}+\beta_{\theta} \Theta_{1}-\left(\Phi_{1}, \nabla\right) v-(v, \nabla) v-(v, \nabla) \Phi_{1}\right), \\
\Theta_{1}=\left(L_{02}\right)^{-1} g_{\theta}-\left(L_{02}\right)^{-1}\left((v, \nabla) \Theta_{1}+\left(\Phi_{1}, \nabla\right) \Theta_{1}+(v, \nabla) \Phi_{2}\right), \\
C_{1}=\left(L_{03}\right)^{-1} g_{c}+\left(L_{03}\right)^{-1}\left(-(v, \nabla) C_{1}-\left(\Phi_{1}, \nabla\right) C_{1}-(v, \nabla) \Phi_{3}+\sum_{j=1}^{m} f_{j} q_{j}\right) .
\end{gathered}
$$


Здесь оператор $\left(L_{01}\right)^{-1}$ отображает элемент $g \in L_{q}\left(Q^{\gamma}\right)$ в пару $(v, p)$, являющуюся решением уравнения $L_{01}(v, p)=g$ и удовлетворяющую условиям

$$
\operatorname{div} v=0, \quad v \in W_{q}^{2,1}\left(Q^{\gamma}\right), \quad p \in L_{q}\left(0, \gamma ; \dot{W}_{q}^{1}(G)\right),
$$

где вектор-функция $v$ удовлетворяет однородным начальным и краевым условиям. Операторы $\left(L_{0 i}\right)^{-1}(i=2,3)$ определяются аналогично с использованием теоремы 2 . Пусть $\left(L_{01}\right)^{-1} g=\left(v_{0}, p_{0}\right)$. Определим пространство $H^{\gamma}$, содержащее векторы $(v, p, \Theta, C)$, где $v \in W_{q}^{2,1}\left(Q^{\gamma}\right)$ - соленоидальная вектор-функция длины $n$, удовлетворяющая однородным условиям (4), $C, \Theta \in W_{q}^{2,1}\left(Q^{\gamma}\right)-$ вектор длины $h$ и скалярная функция, удовлетворяющие однородным условиям (5), $p$ - скалярная функция из $L_{q}\left(0, \gamma ; \dot{W}_{q}^{1}(G)\right)$. Введем в этом пространстве норму

$$
\|(v, p, \Theta, C)\|_{H^{\gamma}}=\|v\|_{W_{q}^{2,1}\left(Q^{\gamma}\right)}+\|p\|_{L_{q}\left(0, \gamma ; \dot{W}_{q}^{1}(G)\right)}+\|\Theta\|_{W_{q}^{2,1}\left(Q^{\gamma}\right)}+\|C\|_{W_{q}^{2,1}\left(Q^{\gamma}\right)} .
$$

Пусть $\boldsymbol{q}=\left(q_{1}, q_{2}, \ldots, q_{s}\right) \in B_{\gamma}=\left\{\boldsymbol{q} \in L_{p}(0, \gamma):\|\boldsymbol{q}\|_{L_{p}(0, \gamma)} \leqslant R_{0}\right\}$. Параметр $R_{0}$ мы уточним ниже. Имеем очевидную оценку (см. теорему 3 и условия (21) на функции $f_{j}$ )

$$
\left\|\left(L_{03}\right)^{-1} \sum_{j=1}^{m} f_{j} q_{j}\right\|_{W_{p}^{2,1}\left(Q^{\gamma}\right)} \leqslant c_{1}\left\|\sum_{j=1}^{m} f_{j} q_{j}\right\|_{L_{p}\left(Q^{\gamma}\right)} \leqslant c_{2}\|\boldsymbol{q}\|_{L_{p}(0, \gamma)} \leqslant c_{2} R_{0} .
$$

Положим

$$
R=3\left(\left\|v_{0}, p_{0},\left(L_{02}\right)^{-1} g_{\theta},\left(L_{03}\right)^{-1} g_{c}\right\|_{H^{T}}+c_{2} R_{0}\right) .
$$

Будем искать решение системы (33)-(35) при данном фиксированном $\boldsymbol{q} \in B_{\gamma}$ :

$$
\omega=A(\omega, \boldsymbol{q}), \quad \omega=(v, p, \Theta, C), \quad \boldsymbol{q}=\left(q_{1}, q_{2}, \ldots, q_{s}\right),
$$

где оператор $A$ определен правой частью системы (33)-(35). Будем считать, что

$$
\omega \in B_{R, \gamma}=\left\{\omega \in H^{\gamma}:\|\omega\|_{H^{\gamma}} \leqslant R\right\} .
$$

Оценим $\|A(\omega, q)\|_{H^{\gamma}}$. Из теорем 1,3 , определения величины $R$ и (36) имеем

$$
\begin{aligned}
& \|A(\omega, \boldsymbol{q})\|_{H^{\gamma}} \leqslant \frac{R}{3}+c\left(\left\|\beta_{c} C_{1}+\beta_{\theta} \Theta_{1}-\left(\Phi_{1}, \nabla\right) v-(v, \nabla) v-(v, \nabla) \Phi_{1}\right\|_{L_{q}\left(Q^{\gamma}\right)}+\right. \\
& \left.\left.\quad+\|(v, \nabla) \Theta_{1}+\left(\Phi_{1}, \nabla\right) \Theta_{1}+(v, \nabla) \Phi_{2}\right)\left\|_{L_{q}\left(Q^{\gamma}\right)}+\right\|(v, \nabla) C_{1}+\left(\Phi_{1}, \nabla\right) C_{1}+(v, \nabla) \Phi_{3} \|_{L_{q}\left(Q^{\gamma}\right)}\right) .
\end{aligned}
$$

Оценим по отдельности каждое из слагаемых, используя лемму 2 :

$$
\left\|\beta_{c} C_{1}\right\|_{L_{q}\left(Q^{\gamma}\right)} \leqslant\left\|\beta_{c}\right\|_{L_{q}(Q)}\left\|C_{1}\right\|_{L_{\infty}\left(Q^{\gamma}\right)} \leqslant c_{1} \gamma^{\beta_{1}}\left\|C_{1}\right\|_{W_{q}^{2,1}\left(Q^{\gamma}\right)} .
$$

Аналогично имеем

Оценим следующее слагаемое:

$$
\left\|\beta_{\theta} \Theta_{1}\right\|_{L_{q}\left(Q^{\gamma}\right)} \leqslant c_{2} \gamma^{\beta_{2}}\left\|\Theta_{1}\right\|_{W_{q}^{2,1}\left(Q^{\gamma}\right)} .
$$

$$
\left\|\left(\Phi_{1}, \nabla\right) v\right\|_{L_{q}\left(Q^{\gamma}\right)} \leqslant\left\|\Phi_{1}\right\|_{L_{q}\left(Q^{T}\right)}\|\nabla v\|_{L_{\infty}\left(Q^{\gamma}\right)} \leqslant c_{3} \gamma^{\beta_{3}}\|v\|_{W_{q}^{2,1}\left(Q^{\gamma}\right)} .
$$

Аналогично получим

$$
\left\|\left(\Phi_{1}, \nabla\right) \Theta_{1}\right\|_{L_{q}\left(Q^{\gamma}\right)} \leqslant c_{4} \gamma^{\beta_{4}}\left\|\Theta_{1}\right\|_{W_{q}^{2,1}\left(Q^{\gamma}\right)}, \quad\left\|\left(\Phi_{1}, \nabla\right) C_{1}\right\|_{L_{q}\left(Q^{\gamma}\right)} \leqslant c_{5} \gamma^{\beta_{5}}\left\|C_{1}\right\|_{W_{q}^{2,1}\left(Q^{\gamma}\right)} .
$$

Имеем

Кроме того,

$$
\left\|(v, \nabla) \Phi_{1}\right\|_{L_{q}\left(Q^{\gamma}\right)} \leqslant\left\|\nabla \Phi_{1}\right\|_{L_{q}\left(Q^{T}\right)}\|v\|_{C\left(Q^{\gamma}\right)} \leqslant c_{6} \gamma^{\beta_{6}}\|v\|_{W_{q}^{2,1}\left(Q^{\gamma}\right)} .
$$

$$
\left\|(v, \nabla) \Phi_{2}\right\|_{L_{q}\left(Q^{\gamma}\right)} \leqslant c_{7} \gamma^{\beta_{7}}\|v\|_{W_{q}^{2,1}\left(Q^{\gamma}\right)}, \quad\left\|(v, \nabla) \Phi_{3}\right\|_{L_{q}\left(Q^{\gamma}\right)} \leqslant c_{8} \gamma^{\beta_{8}}\|v\|_{W_{q}^{2,1}\left(Q^{\gamma}\right)} .
$$

Наконец,

$$
\|(v, \nabla) v\|_{L_{q}\left(Q^{\gamma}\right)} \leqslant\|\nabla v\|_{C\left(Q^{\gamma}\right)}\|v\|_{C\left(Q^{\gamma}\right)} \leqslant c_{9}(R) \gamma^{\beta_{9}}\|v\|_{W_{q}^{2,1}\left(Q^{\gamma}\right)},
$$


Аналогично,

Суммируя, получим

$$
\begin{gathered}
\left\|(v, \nabla) \Theta_{1}\right\|_{L_{q}\left(Q^{\gamma}\right)} \leqslant c_{10}(R) \gamma^{\beta_{10}}\left\|\Theta_{1}\right\|_{W_{q}^{2,1}\left(Q^{\gamma}\right)}, \\
\left\|(v, \nabla) C_{1}\right\|_{L_{q}\left(Q^{\gamma}\right)} \leqslant c_{11}(R) \gamma^{\beta_{11}}\left\|C_{1}\right\|_{W_{q}^{2,1}\left(Q^{\gamma}\right)}
\end{gathered}
$$

$$
\|A(\omega, \boldsymbol{q})\|_{H^{\gamma}} \leqslant \frac{R}{3}+c_{1}(R) \gamma^{\beta_{1}}\|v\|_{W_{q}^{2,1}\left(Q^{\gamma}\right)}+c_{2}(R) \gamma^{\beta_{2}}\left\|\Theta_{1}\right\|_{W_{q}^{2,1}\left(Q^{\gamma}\right)}+c_{3}(R) \gamma^{\beta_{3}}\left\|C_{1}\right\|_{W_{q}^{2,1}\left(Q^{\gamma}\right)} .
$$

Так как $\gamma \in(0, T]$, то, выбрав $\beta=\min \left(\beta_{1}, \beta_{2}, \beta_{3}\right)$, имеем

$$
\|A(\omega, \boldsymbol{q})\|_{H^{\gamma}} \leqslant \frac{R}{3}+c_{0}(R) \gamma^{\beta}\|\omega\|_{H^{\gamma}}
$$

где постоянная $c_{1}$ не зависит от $R$ и $\beta$. Выберем такую константу $\gamma_{0} \leqslant T$, что $c_{0}(R) \gamma^{\beta} \leqslant R / 3$ для всех $\gamma \leqslant \gamma_{0}$. Тогда неравенство (38) можно переписать в виде

$$
\|A(\omega, \boldsymbol{q})\|_{H^{\gamma}} \leqslant R \quad \forall \omega \in B_{R, \gamma}, \boldsymbol{q} \in B_{\gamma} .
$$

Это означает, что для каждого $\boldsymbol{q} \in B_{\gamma}$ оператор $A(\omega, \boldsymbol{q})$ переводит шар $B_{R, \gamma}$ в себя. Аналогично рассуждая, можем получить оценку для $\left\|A\left(\omega^{1}, \boldsymbol{q}\right)-A\left(\omega^{2}, \boldsymbol{q}\right)\right\|_{H^{\gamma}}$, где $\omega^{i}=\left(v^{i}, p^{i}, \Theta^{i}, C^{i}\right), i=1,2$. Имеем

$$
\begin{gathered}
\left\|A\left(\omega^{1}, \boldsymbol{q}\right)-A\left(\omega^{2}, \boldsymbol{q}\right)\right\|_{H^{\gamma}} \leqslant c\left(\| \beta_{c}\left(C^{1}-C^{2}\right)+\beta_{\theta}\left(\Theta^{1}-\Theta^{2}\right)-\left(\Phi_{1}, \nabla\right)\left(v^{1}-v^{2}\right)-\left(v^{1}, \nabla\right) v^{1}+\right. \\
+\left(v^{2}, \nabla\right) v^{2}-\left(v^{1}-v^{2}, \nabla\right) \Phi_{1} \|_{L_{q}\left(Q^{\gamma}\right)}+ \\
+\left\|\left(\left(v^{1}, \nabla\right) \Theta^{1}-\left(v^{2}, \nabla\right) \Theta^{2}\right)+\left(\Phi_{1}, \nabla\right)\left(\Theta^{1}-\Theta^{2}\right)+\left(v^{1}-v^{2}, \nabla\right) \Phi_{2}\right\|_{L_{q}\left(Q^{\gamma}\right)}+ \\
\left.+\left\|\left(v^{1}, \nabla\right) C^{1}-\left(v^{2}, \nabla\right) C^{2}+\left(\Phi_{1}, \nabla\right)\left(C^{1}-C^{2}\right)+\left(v^{1}-v^{2}, \nabla\right) \Phi_{3}\right\|_{L_{q}\left(Q^{\gamma}\right)}\right) .
\end{gathered}
$$

Используя лемму 2, оценим по отдельности каждое из слагаемых:

$$
\begin{gathered}
\left\|\beta_{c}\left(C^{1}-C^{2}\right)\right\|_{L_{q}\left(Q^{\gamma}\right)} \leqslant c_{1} \gamma^{\beta_{1}}\left\|\left(C^{1}-C^{2}\right)\right\|_{W_{q}^{2,1}\left(Q^{\gamma}\right)}, \\
\left\|\beta_{\theta}\left(\Theta^{1}-\Theta^{2}\right)\right\|_{L_{q}\left(Q^{\gamma}\right)} \leqslant c_{2} \gamma^{\beta_{2}}\left\|\left(\Theta^{1}-\Theta^{2}\right)\right\|_{W_{q}^{2,1}\left(Q^{\gamma}\right)}
\end{gathered}
$$

Кроме того, имеем

$$
\left\|\left(\Phi_{1}, \nabla\right)\left(v^{1}-v^{2}\right)\right\|_{L_{q}\left(Q^{\gamma}\right)} \leqslant\left\|\Phi_{1}\right\|_{L_{q}\left(Q^{T}\right)}\left\|\nabla\left(v^{1}-v^{2}\right)\right\|_{C\left(\overline{Q^{\gamma}}\right)} \leqslant c_{3} \gamma^{\beta_{3}}\left\|v^{1}-v^{2}\right\|_{W_{q}^{2,1}\left(Q^{\gamma}\right)} .
$$

Аналогично можно оценить

$$
\begin{aligned}
& \left\|\left(\Phi_{1}, \nabla\right)\left(\Theta^{1}-\Theta^{2}\right)\right\|_{L_{q}\left(Q^{\gamma}\right)} \leqslant c_{4} \gamma^{\beta_{4}}\left\|\Theta^{1}-\Theta^{2}\right\|_{W_{q}^{2,1}\left(Q^{\gamma}\right)} \\
& \left\|\left(\Phi_{1}, \nabla\right)\left(C^{1}-C^{2}\right)\right\|_{L_{q}\left(Q^{\gamma}\right)} \leqslant c_{5} \gamma^{\beta_{5}}\left\|C^{1}-C^{2}\right\|_{W_{q}^{2,1}\left(Q^{\gamma}\right)}
\end{aligned}
$$

Оценим следующее слагаемое:

$$
\left\|\left(v^{1}-v^{2}, \nabla\right) \Phi_{1}\right\|_{L_{q}\left(Q^{\gamma}\right)} \leqslant\left\|\nabla \Phi_{1}\right\|_{L_{q}\left(Q^{T}\right)}\left\|v^{1}-v^{2}\right\|_{C\left(\overline{Q^{\gamma}}\right)} \leqslant c_{6} \gamma^{\beta_{6}}\left\|v^{1}-v^{2}\right\|_{W_{q}^{2,1}\left(Q^{\gamma}\right)} .
$$

Аналогично получим

Учитывая, что

$$
\begin{gathered}
\left\|\left(v^{1}-v^{2}, \nabla\right) \Phi_{2}\right\|_{L_{q}\left(Q^{\gamma}\right)} \leqslant c_{7} \gamma^{\beta_{7}}\left\|v^{1}-v^{2}\right\|_{W_{q}^{2,1}\left(Q^{\gamma}\right)} \\
\left\|\left(v^{1}-v^{2}, \nabla\right) \Phi_{3}\right\|_{L_{q}\left(Q^{\gamma}\right)} \leqslant c_{8} \gamma^{\beta 8}\left\|v^{1}-v^{2}\right\|_{W_{q}^{2,1}\left(Q^{\gamma}\right)}
\end{gathered}
$$

$$
\left(v^{1}, \nabla\right) v^{1}-\left(v^{2}, \nabla\right) v^{2}=\left(v^{1}-v^{2}, \nabla\right) v^{1}+\left(v^{2}, \nabla\right)\left(v^{1}-v^{2}\right),
$$


находим

$$
\begin{aligned}
\left\|\left(v^{1}-v^{2}, \nabla\right) v^{1}\right\|_{L_{q}\left(Q^{\gamma}\right)} & \leqslant\left\|\nabla v^{1}\right\|_{C\left(Q^{\gamma}\right)}\left\|v^{1}-v^{2}\right\|_{C\left(Q^{\gamma}\right)} \leqslant c(R) \gamma^{\beta}\left\|v^{1}-v^{2}\right\|_{W_{q}^{2,1}\left(Q^{\gamma}\right)}, \\
\left\|\left(v^{2}, \nabla\right)\left(v^{1}-v^{2}\right)\right\|_{L_{q}\left(Q^{\gamma}\right)} & \leqslant\left\|v^{2}\right\|_{C\left(Q^{\gamma}\right)}\left\|\nabla\left(v^{1}-v^{2}\right)\right\|_{C\left(Q^{\gamma}\right)} \leqslant c(R) \gamma^{\beta}\left\|v^{1}-v^{2}\right\|_{W_{q}^{2,1}\left(Q^{\gamma}\right)}
\end{aligned}
$$

Далее имеем

$$
\left\|\left(v^{1}, \nabla\right) v^{1}-\left(v^{2}, \nabla\right) v^{2}\right\|_{L_{q}\left(Q^{\gamma}\right)} \leqslant c_{9}(R) \gamma^{\beta_{9}}\left\|v^{1}-v^{2}\right\|_{W_{q}^{2,1}\left(Q^{\gamma}\right)}
$$

Аналогично,

$$
\begin{aligned}
& \left\|\left(v^{1}, \nabla\right) \Theta_{1}^{1}-\left(v^{2}, \nabla\right) \Theta_{1}^{2}\right\|_{L_{q}\left(Q^{\gamma}\right)} \leqslant c_{10}(R) \gamma^{\beta_{10}}\left\|\Theta_{1}^{1}-\Theta_{1}^{2}\right\|_{W_{q}^{2,1}\left(Q^{\gamma}\right)} \\
& \left\|\left(v^{1}, \nabla\right) C^{1}-\left(v^{2}, \nabla\right) C^{2}\right\|_{L_{q}\left(Q^{\gamma}\right)} \leqslant c_{11}(R) \gamma^{\beta_{11}}\left\|C^{1}-C^{2}\right\|_{W_{q}^{2,1}\left(Q^{\gamma}\right)}
\end{aligned}
$$

Выбрав соответствующее $\beta$, окончательно имеем:

$$
\left\|A\left(\omega^{1}, \boldsymbol{q}\right)-A\left(\omega^{2}, \boldsymbol{q}\right)\right\|_{H^{\gamma}} \leqslant c_{4}(R) \gamma^{\beta}\left\|\omega^{1}-\omega^{2}\right\|_{H^{\gamma}}
$$

что означает, что оператор $A$ является сжимающим при $c_{4}(R) \gamma^{\beta}=r_{0}<1$. В частности, имеем

$$
\left\|A\left(\omega^{1}, \boldsymbol{q}\right)-A(0, \boldsymbol{q})\right\|_{H^{\gamma}} \leqslant c_{4}(R) \gamma^{\beta}\left\|\omega^{1}\right\|_{H^{\gamma}} .
$$

Зафиксируем константу $r_{0}<1$ и найдем такую постоянную $\gamma_{1} \leqslant \gamma_{0}$, что $c_{4}(R) \gamma^{\beta} \leqslant r_{0}$ для $\gamma \leqslant \gamma_{1}$. Как прямое следствие теоремы о неподвижной точке, имеем, что для любого $\boldsymbol{q} \in B_{\gamma}$ при $\gamma \leqslant \gamma_{1}$ система (37) и система (33)-(35) соответственно имеет единственное решение $\omega$ в шаре $B_{R, \gamma}$. Решение удовлетворяет неравенству

$$
\|\omega\|_{H^{\gamma}} \leqslant\|A(0, \boldsymbol{q})\|_{H^{\gamma}}+\|A(\omega, \boldsymbol{q})-A(0, \boldsymbol{q})\|_{H^{\gamma}} \leqslant \frac{R}{3}+r_{0}\|\omega\|_{H^{\gamma}}
$$

Это неравенство означает, что

$$
\|\omega\|_{H^{\gamma}} \leqslant \frac{R}{3\left(1-r_{0}\right)}
$$

Рассмотрим два вектора $\boldsymbol{q}^{1}, \boldsymbol{q}^{2} \in B_{\gamma}$ и найдем два решения $\omega^{1}, \omega^{2}\left(\omega^{i}=\left(v^{i}, p^{i}, \Theta^{i}, C^{i}\right), i=1,2\right)$ системы (33)-(35). Их разность $\omega^{1}-\omega^{2}$ удовлетворяет равенству

$$
\omega^{1}-\omega^{2}=A\left(\omega^{1}, \boldsymbol{q}^{1}\right)-A\left(\omega^{2}, \boldsymbol{q}^{1}\right)+A\left(\omega^{2}, \boldsymbol{q}^{1}\right)-A\left(\omega^{2}, \boldsymbol{q}^{2}\right),
$$

из которого следует

$$
\left\|\omega^{1}-\omega^{2}\right\|_{H^{\gamma}} \leqslant r_{0}\left\|\omega^{1}-\omega^{2}\right\|_{H^{\gamma}}+c_{1}\left\|\boldsymbol{q}^{1}-\boldsymbol{q}^{2}\right\|_{L_{q}(0, \gamma)}
$$

и

$$
\left\|\omega^{1}-\omega^{2}\right\|_{H^{\gamma}} \leqslant \frac{c_{1}}{1-r_{0}}\left\|\boldsymbol{q}^{1}-\boldsymbol{q}^{2}\right\|_{L_{q}(0, \gamma)}
$$

Пусть $\gamma \leqslant \gamma_{1}$. Умножим уравнение (31) скалярно на вектор $\varphi_{k}$ и проинтегрируем результат по $G$, считая, что условия (32) выполнены. Получаем равенство

$$
\begin{aligned}
\tilde{\psi}_{k}^{\prime}-\int_{G} & \left(\left(\sum_{i, j=1}^{n} a_{i j} C_{1 x_{i} x_{j}}+\sum_{j=1}^{n} a_{j} C_{1 x_{j}}+a_{0} C_{1}\right), \varphi_{k}(x)\right) d x= \\
& =\left(g_{c}, \varphi_{k}\right)_{0}-\int_{G}\left(\left((v, \nabla) C_{1}+\left(\Phi_{1}, \nabla\right) C_{1}+(v, \nabla) \Phi_{3}\right), \varphi_{k}\right) d x+\sum_{j=1}^{s} q_{j} \int_{G}\left(f_{j}, \varphi_{k}\right) d x
\end{aligned}
$$

где

$$
\left(g_{c}, \varphi_{k}\right)_{0}=\int_{G}\left(g_{c}, \varphi_{k}\right) d x .
$$

Это равенство можно переписать в виде

$$
\boldsymbol{q}=B^{-1} S_{0}(\boldsymbol{q})=S(\boldsymbol{q}),
$$


где $k$-я координата $s_{k}$ вектора $S_{0}$ записывается в виде

$$
\begin{aligned}
\alpha_{k}(\boldsymbol{q})=-\int_{G}\left(\left(\sum_{i, j=1}^{n} a_{i j} C_{1 x_{i} x_{j}}+\sum_{j=1}^{n} a_{j} C_{1 x_{j}}\right.\right. & \left.\left.+a_{0} C_{1}\right), \varphi_{k}(x)\right) d x+ \\
& \left.+\int_{G}\left((v, \nabla) C_{1}+\left(\Phi_{1}, \nabla\right) C_{1}+(v, \nabla) \Phi_{3}\right), \varphi_{k}\right) d x \\
s_{k}=F_{k}+\alpha_{k}(\boldsymbol{q}), \quad F_{k} & =\tilde{\psi}_{k}^{\prime}-\left(g_{c}, \varphi_{k}\right)_{0},
\end{aligned}
$$

Вектор-функции $C_{1}, v$, входящие в выражение $S(\boldsymbol{q})$, выражаются через вектор $\boldsymbol{q}$ через решение системы (37). Выберем параметр $R_{0}$ и соответственно параметр $R$. Положим $F=\left(F_{1}, F_{2}, \ldots, F_{n}\right)$ и определим $R_{0}=2\left\|B^{-1} F\right\|_{L_{q}(0, T)}$. Тогда параметр $R$, определенный выше, вычисляется через величину $R_{0}$ и нормы исходных данных. По параметру $R$ мы определили величину $\gamma_{1}$. Величина $\gamma_{1}$ будет зависеть только от норм данных. Тогда при всех $\gamma \leqslant \gamma_{1}$ оператор $S$ определен корректно. Докажем разрешимость уравнения (43). Пусть $\boldsymbol{q}^{1}, \boldsymbol{q}^{2} \in B_{\gamma}$ с $\gamma \leqslant \gamma_{1}$. Приведем необходимые оценки и покажем, что оператор $S$ переводит шар $B_{\gamma}$ в себя и является в нем сжимающим, если параметр $\gamma$ мал. Основные слагаемые, входящие в функцию $\alpha_{k}$, имеют вид

$$
J_{k}=\int_{G}\left(a_{i j} C_{1 x_{i} x_{j}}, \varphi_{k}(x)\right) d x .
$$

Возьмем $\varepsilon<\min \left(1 / r, \varepsilon_{0}\right)$ (см. условие $\left.(19)\right)$. Пусть символ $\stackrel{\circ}{W} \underset{r}{s}(G)(s \leqslant 1)$ обозначает пространство функций из $W_{r}^{s}(G)$, обращающихся в нуль на $\Gamma$ при $s>1 / r$, a $W_{p}^{-s}(G)$ - двойственное пространство. В силу двойственности (неравенство Шварца) имеем

$$
\left|J_{k}\right| \leqslant\left\|C_{1 x_{i} x_{j}}\right\|_{W_{q}^{-\varepsilon}(G)}\left\|a_{i j}^{*} \varphi_{k}\right\|_{W_{r}^{\varepsilon}(G)}, \quad \frac{1}{r}+\frac{1}{q}=1,
$$

где $a_{i j}^{*}$-сопряженная матрица. Ее элементы (см. условие (19)) принадлежат классу $C\left([0, T] ; C^{\varepsilon_{0}}(\bar{G})\right)$. Тогда в соответствии с теоремой о точечных мультипликаторах (см. [55, теорема 3.3 .2$, с. 198]; можно также использовать определение нормы) имеем

$$
\left\|a_{i j}^{*} \varphi_{k}\right\|_{\stackrel{\circ}{r}_{r}^{\varepsilon}(G)} \leqslant c\left\|\varphi_{k}\right\|_{\stackrel{\circ}{r}_{r}^{\varepsilon}(G)} \leqslant c_{1},
$$

где $c, c_{1}$ - некоторые постоянные, не зависящие от времени. Отметим, что $\stackrel{\circ}{{ }^{\varepsilon}} r(G)=W_{r}^{s}(G)$ при $s<1 / r$, поэтому правая часть предыдущего неравенства конечна. С другой стороны, имеет место неравенство

$$
\left\|C_{1 x_{i} x_{j}}\right\|_{W_{q}^{-\varepsilon}(G)} \leqslant c\left\|C_{1}\right\|_{W_{q}^{2-\varepsilon}(G)} .
$$

Неравенства такого вида возникают часто. Они вытекают из интерполяционных свойств линейных операторов. Поясним доказательство. Рассмотрим отображение $M: v \rightarrow v_{x_{j}}$. Очевидно, $M \in L\left(L_{q}(G), W_{q}^{-1}(G)\right) \cap L\left(W_{q}^{1}(G), L_{q}(G)\right)$. Следовательно,

Однако

$$
M \in L\left(\left(L_{q}(G), W_{q}^{-1}(G)\right)_{\theta, q},\left(W_{q}^{1}(G), L_{q}(G)\right)_{\theta, q}\right), \quad \theta \in(0,1) .
$$

$$
\left(W_{q}^{1}(G), L_{q}(G)\right)_{\theta, q}=W_{q}^{1-\theta}(G), \quad\left(L_{q}(G), W_{q}^{-1}(G)\right)_{\theta, q}=W_{q}^{-\theta}(G)
$$

(см. [54]). Таким образом, имеет место неравенство

$$
\left\|v_{x_{j}}\right\|_{W_{q}^{-\varepsilon}(G)} \leqslant c\|v\|_{W_{q}^{1-\varepsilon}(G)} .
$$

Отсюда и вытекает неравенство (44). Из полученных неравенств вытекает оценка

$$
\left\|J_{k}\right\|_{L_{q}(0, \gamma)} \leqslant c\left\|C_{1}\right\|_{L_{q}\left(0, \gamma ; W_{q}^{2-\varepsilon}(G)\right)}
$$

Далее используем интерполяционные неравенства (см. [54]) и лемму 1. Получаем

$$
\left\|J_{k}\right\|_{L_{q}(0, \gamma)} \leqslant c\left\|C_{1}\right\|_{L_{q}\left(0, \gamma ; W_{q}^{2-\varepsilon}(G)\right)} \leqslant c_{1}\left\|C_{1}\right\|_{L_{q}\left(Q^{\gamma}\right)}^{\varepsilon}\left\|C_{1}\right\|_{L_{q}\left(0, \gamma ; W_{q}^{2}(G)\right)}^{2-\varepsilon} \leqslant c_{2} \gamma^{\varepsilon}\left\|C_{1}\right\|_{W_{q}^{2,1}\left(Q^{\gamma}\right)}
$$


Оставшиеся слагаемые в выражении $\alpha_{k}$ оцениваются гораздо проще. Рассмотрим, например, выражение

$$
I_{k}=\int_{G}\left(a_{j} C_{1 x_{j}}, \varphi_{k}(x)\right) d x .
$$

Имеем

$$
\left|I_{k}\right| \leqslant c\left\|\varphi_{k}(x)\right\|_{L_{r}(G)}\left\|a_{j} C_{1 x_{j}}\right\|_{L_{q}(G)} \leqslant c_{1}\left\|a_{j}\right\|_{L_{q}(G)}\left\|C_{1 x_{j}}\right\|_{L_{\infty}(Q)} .
$$

Далее, обращаясь к лемме 2 получим неравенство

$$
\left\|I_{k}\right\|_{L_{q}(0, \gamma)} \leqslant c_{2} \gamma^{\beta}\left\|C_{1}\right\|_{W_{q}^{2,1}\left(Q^{\gamma}\right)},
$$

где $\beta$ - некоторая положительная постоянная. Оставшиеся слагаемые в выражении $\alpha_{k}(\boldsymbol{q})$ оцениваются аналогично. Положим $\boldsymbol{\alpha}=\left(\alpha_{1}, \alpha_{2}, \ldots, \alpha_{s}\right)^{T}$. Таким образом, можем сказать, что найдется такое число $\beta_{0}>0$, что имеет место неравенство

$$
\left\|B^{-1} \boldsymbol{\alpha}(\boldsymbol{q})\right\|_{L_{q}(0, \gamma)} \leqslant c \gamma^{\beta_{0}}\left(\left\|C_{1}\right\|_{W_{q}^{2,1}\left(Q^{\gamma}\right)}+\|v\|_{W_{q}^{2,1}\left(Q^{\gamma}\right)}\right) \leqslant C(R) \gamma^{\beta_{0}} .
$$

Теперь возьмем, два различных вектора $\boldsymbol{q}^{1}, \boldsymbol{q}^{2}$. Запишем разность $S_{0}\left(\boldsymbol{q}^{1}\right)-S_{0}\left(\boldsymbol{q}^{2}\right)$. Обозначим через $C^{i}, v^{i}$ (где $\left.v^{i}=\left(v_{1}^{i}, v_{2}^{i}, \ldots, v_{n}^{i}\right), i=1,2\right)$ решения системы $(37)$, соответствующие этим векторам. Отметим, что данные векторы входят в функции $\alpha_{k}$ почти линейно. Поэтому легко увидеть, что будет справедлива аналогичная оценка, но в правой части уже будут нормы разностей $C^{1}-C^{2}, v^{1}-v^{2}$. Используя (41), получим

$$
\left\|B^{-1} \boldsymbol{\alpha}\left(\boldsymbol{q}^{1}\right)-B^{-1} \boldsymbol{\alpha}\left(\boldsymbol{q}^{2}\right)\right\|_{L_{q}(0, \gamma)} \leqslant c(R) \gamma^{\beta_{1}}\left\|\boldsymbol{q}^{1}-\boldsymbol{q}^{2}\right\|_{L_{q}(0, \gamma)},
$$

где $\beta_{1}$ - некоторая положительная постоянная. Используя определение величины $R_{0}$ имеем неравенство

$$
\|S(\boldsymbol{q})\| \leqslant R_{0} / 2+C(R) \gamma^{\beta_{0}}, \quad\left\|S\left(\boldsymbol{q}^{1}\right)-S\left(\boldsymbol{q}^{2}\right)\right\|_{L_{q}(0, \gamma)} \leqslant c(R) \gamma^{\beta_{1}}\left\|\boldsymbol{q}^{1}-\boldsymbol{q}^{2}\right\|_{L_{q}(0, \gamma)} .
$$

Остается выбрать такую постоянную $\gamma_{2} \leqslant \gamma_{1}$, что если $\gamma \leqslant \gamma_{2}$, то $C(R) \gamma^{\beta_{0}} \leqslant R_{0} / 2$ и $c(R) \gamma^{\beta_{1}} \leqslant 1 / 2$. Тогда при $\gamma \leqslant \gamma_{2}$ уравнение (43) имеет единственное решение в шаре $B_{\gamma}$. Векторы $C_{1}, v$ и функцию $\Theta_{1}$ находим как решение системы (37). Покажем, что условия переопределения для функции $C_{1}$ выполняются. Умножим уравнение (31) скалярно на вектор-функцию $\varphi_{k}$ и проинтегрируем результат по $G$. Вычитая полученное равенство из $k$-го уравнения в $(42)$, получим равенство

$$
\int_{G}\left(C_{1 t}, \varphi_{k}\right) d x=\tilde{\psi}_{k},
$$

а это как раз и есть наше условие переопределения. Таким образом, мы построили решение нашей задачи локально по времени.

Рассмотрим вопрос об устойчивости решений. Фиксируем шар радиуса $R$ в пространстве данных. В силу выбора шара, в котором мы ищем вектор $\boldsymbol{q}$ и решения $C_{1}, v, \Theta_{1}$, можем считать, что величина $R_{0}$ фиксирована и соответственно величина $R$, введенная в доказательстве, также фиксирована. Они определяются радиусом шара, в котором расположены данные задачи. Тогда, повторяя рассуждения, получим, что можно взять параметр $\gamma_{2}$ одним и тем же для всех данных из нашего класса. Будут справедливы оценки (46), (41), (40). Возьмем два решения, отвечающие двум различным наборам данных $\left(C^{i}, \Theta^{i}, v^{i}, q^{i}\right)$, где $v^{i}=\left(v_{1}^{i}, v_{2}^{i}, \ldots, v_{n}^{i}\right), i=1,2$. Каждое из них удовлетворяет системе (33)-(35), где справа вместо функций $g, g_{\theta}, g_{0 c}$ стоят соответствующие функции $g^{i}, g_{\theta}^{i}, g_{0 c}^{i}$. Вычитая эти две системы друг из друга, можем оценить норму разности решений, и вместо оценки (41) получим оценку вида

$$
\left\|\omega^{1}-\omega^{2}\right\|_{H^{\gamma}} \leqslant c_{1}\left(\left\|q^{1}-q^{2}\right\|_{L_{q}(0, \gamma)}+\left\|g^{1}-g^{2}\right\|_{L_{q}\left(Q^{\gamma}\right)}+\left\|g_{\theta}^{1}-g_{\theta}^{2}\right\|_{L_{q}\left(Q^{\gamma}\right)}+\left\|g_{0 c}^{1}-g_{0 c}^{2}\right\|_{L_{q}\left(Q^{\gamma}\right)}\right) .
$$

Далее рассмотрим равенства (43), записанные для этих двух решений. Вычитая их друг из друга и повторяя использованные выше рассуждения, придем к оценкам

$$
\left\|q^{1}-q^{2}\right\|_{L_{q}(0, \gamma)} \leqslant c(R) \gamma^{\beta}\left\|\omega^{1}-\omega^{2}\right\|_{H^{\gamma}}+\left\|\boldsymbol{F}_{1}-\boldsymbol{F}_{2}\right\|_{L_{q}(0, \gamma)},
$$


где $\beta>0, c(R)$ - положительные постоянные и векторы $\boldsymbol{F}_{1}, \boldsymbol{F}_{2}$ - определенный выше вектор $\boldsymbol{F}$, записанный для двух наборов данных. Выбирая такое $\gamma_{3} \leqslant \gamma_{2}$, что $c_{1} c(R) \gamma^{\beta} \leqslant 1 / 2$ при $\gamma \leqslant \gamma_{3}$, и используя неравенство (49) в правой части (48), придем к неравенству

$$
\left\|\omega^{1}-\omega^{2}\right\|_{H^{\gamma}} \leqslant c_{2}\left(\left\|g^{1}-g^{2}\right\|_{L_{q}\left(Q^{\gamma}\right)}+\left\|g_{\theta}^{1}-g_{\theta}^{2}\right\|_{L_{q}\left(Q^{\gamma}\right)}+\left\|g_{0 c}^{1}-g_{0 c}^{2}\right\|_{L_{q}\left(Q^{\gamma}\right)}+\left\|\boldsymbol{F}_{1}-\boldsymbol{F}_{2}\right\|_{L_{q}(0, \gamma)}\right),
$$

где $\gamma \leqslant \gamma_{3}$. Используя это неравенство в правой части (49), получим

$$
\left.\left\|q^{1}-q^{2}\right\|_{L_{q}(0, \gamma)} \leqslant c_{3}\left(\left\|g^{1}-g^{2}\right\|_{L_{q}\left(Q^{\gamma}\right)}+\left\|g_{\theta}^{1}-g_{\theta}^{2}\right\|_{L_{q}\left(Q^{\gamma}\right)}+\left\|g_{0 c}^{1}-g_{0 c}^{2}\right\|_{L_{q}\left(Q^{\gamma}\right)}\right)+\left\|\boldsymbol{F}_{1}-\boldsymbol{F}_{2}\right\|_{L_{q}(0, \gamma)}\right) .
$$

Последние две оценки и гарантируют выполнение оценки устойчивости из формулировки теоремы. Теорема доказана.

Доказательство теоремы 5. Доказательство локальной разрешимости практически совпадает с доказательством предыдущей теоремы. Мы повторим только основные моменты. Как и ранее, строим функции $\Phi_{i}, i=1,2,3$, удовлетворяющие начальным и граничным данным, и делаем замену

$$
u=v+\Phi_{1}, \quad \Theta=\Theta_{1}+\Phi_{2}, \quad C=C_{1}+\Phi_{3} .
$$

Получим

$$
\begin{gathered}
L_{01}(v, p)=v_{t}-\nu \Delta v+\nabla p=g+\sum_{j=1}^{n} B_{j} v_{x_{j}}+B_{0} v+\beta_{C} C_{1}+\beta_{\theta} \Theta_{1}, \\
\operatorname{div} v=0 \\
L_{02} \Theta_{1}=\Theta_{1 t}-\lambda_{\theta} \Delta \Theta_{1}+\sum_{j=1}^{n} b_{j} \Theta_{1 x_{j}}+b_{0} \Theta_{1}=g_{\theta}+\sum_{j=1}^{n} b^{j} v_{j}, \\
L_{03} C_{1}=C_{1 t}-\lambda_{C} \Delta C_{1}+\sum_{j=1}^{n} c_{j} C_{1 x_{j}}+c_{0} C_{1}=g_{c}+\sum_{j=1}^{n} c^{j} v_{j}+\sum_{j=1}^{r} f_{j} q_{j},
\end{gathered}
$$

где новые функции $g, g_{\theta}$ и вектор-функция $g_{c}$ имеют вид

$$
\begin{aligned}
& g=f-\Phi_{1 t}+\nu \Delta \Phi_{1}+\sum_{j=1}^{n} B_{j} \Phi_{1 x_{j}}+B_{0} \Phi_{1}+\beta_{C} \Phi_{3}+\beta_{\theta} \Phi_{2}, \\
& g_{\theta}=f_{\theta}-L_{02} \Phi_{2}+\sum_{j=1}^{n} b^{j} \Phi_{1 j}, \quad g_{c}=f_{0}-L_{03} \Phi_{3}+\sum_{j=1}^{n} c^{j} \Phi_{1 j} .
\end{aligned}
$$

Здесь величины $\Phi_{1 j}$ - координаты вектор-функции $\Phi_{1}$. Новые функции $v, \Theta_{1}$ и вектор-функция $C_{1}$ удовлетворяют однородным начальным и граничным условиям, а также выполнены условия переопределения

$$
\int_{G}\left(C_{1}, \varphi_{j}(x)\right) d x=\psi_{j}(t)-\int_{G}\left(\Phi_{3}, \varphi_{j}(x)\right) d x=\tilde{\psi}_{j}(t), \quad i=1,2, \ldots, s .
$$

Мы свели задачу к эквивалентной задаче. Пусть $\gamma \in(0, T]$. Как и в предыдущей теореме, используя теоремы 1,2 , можем переписать уравнения (51)-(53) в следующей форме:

$$
\begin{gathered}
(v, p)=\left(L_{01}\right)^{-1} g+\left(L_{01}\right)^{-1}\left(\sum_{j=1}^{n} B_{j} v_{x_{j}}+B_{0} v+\beta_{C} C_{1}+\beta_{\theta} \Theta_{1}\right) \\
\Theta_{1}=\left(L_{02}\right)^{-1} g_{\theta}-\left(L_{02}\right)^{-1}\left(g_{\theta}+\sum_{j=1}^{n} b^{j} v_{j}\right) \\
C_{1}=\left(L_{03}\right)^{-1} g_{c}+\left(L_{03}\right)^{-1}\left(g_{c}+\sum_{j=1}^{n} c^{j} v_{j}+\sum_{j=1}^{r} f_{j} q_{j}\right) .
\end{gathered}
$$


Пусть $\boldsymbol{q}=\left(q_{1}, q_{2}, \ldots, q_{s}\right) \in B_{\gamma}=\left\{\boldsymbol{q} \in L_{p}(0, \gamma):\|\boldsymbol{q}\|_{L_{p}(0, \gamma)} \leqslant R_{0}\right\}$. Параметр $R_{0}$ мы уточним ниже. Будем искать решение системы (55)-(57) при данном фиксированном $\boldsymbol{q} \in B_{\gamma}$ :

$$
\omega=A(\omega, \boldsymbol{q}), \quad \omega=(v, p, \Theta, C), \quad \boldsymbol{q}=\left(q_{1}, q_{2}, \ldots, q_{s}\right),
$$

где оператор $A$ определен правой частью системы (55)-(57). Будем считать, что

$$
\omega \in B_{R, \gamma}=\left\{\omega \in H^{\gamma}:\|\omega\|_{H^{\gamma}} \leqslant R\right\},
$$

где

$$
R=3\left(\left\|v_{0}, p_{0},\left(L_{02}\right)^{-1} g_{\theta},\left(L_{03}\right)^{-1} g_{c}\right\|_{H^{T}}+c_{2} R_{0}\right) .
$$

Аналоги оценок (38), (39) запишутся в виде

$$
\|A(\omega, \boldsymbol{q})\|_{H^{\gamma}} \leqslant \frac{R}{3}+c_{3} \gamma^{\beta}\|\omega\|_{H^{\gamma}}
$$

где постоянная $c_{3}$ не зависит от $R$ и $\beta$-некоторая положительная постоянная;

$$
\left\|A\left(\omega^{1}, \boldsymbol{q}\right)-A\left(\omega^{2}, \boldsymbol{q}\right)\right\|_{H^{\gamma}} \leqslant c_{4} \gamma^{\beta}\left\|\omega^{1}-\omega^{2}\right\|_{H^{\gamma}},
$$

что означает, что оператор $A$ является сжимающим при $c_{4} \gamma^{\beta}<1$. В частности, имеем

$$
\left\|A\left(\omega^{1}, \boldsymbol{q}\right)-A(0, \boldsymbol{q})\right\|_{H^{\gamma}} \leqslant c_{4} \gamma^{\beta}\left\|\omega^{1}\right\|_{H^{\gamma}} .
$$

Легко видеть, что постоянные $c_{3}$ и $c_{4}$ не зависят от $R$, а зависят от норм коэффициентов в $Q$. Зафиксируем константу $r_{0}<1$ и найдем такую постоянную $\gamma_{1}$, что

$$
c_{4} \gamma^{\beta} \leqslant r_{0}, \quad c_{3} \gamma^{\beta} \leqslant \frac{1}{3}
$$

для $\gamma \leqslant \gamma_{1}$. При помощи теоремы о неподвижной точке заключаем, что для любого $\boldsymbol{q} \in B_{\gamma}$ при $\gamma \leqslant \gamma_{1}$ система (58) и система (55)-(57) имеют единственное решение $\omega$ в шаре $B_{R, \gamma}$. Решение удовлетворяет неравенству

$$
\|\omega\|_{H^{\gamma}} \leqslant\|A(0, \boldsymbol{q})\|_{H^{\gamma}}+\|A(\omega, \boldsymbol{q})-A(0, \boldsymbol{q})\|_{H^{\gamma}} \leqslant \frac{R}{3}+r_{0}\|\omega\|_{H^{\gamma}}
$$

Это неравенство означает, что

$$
\|\omega\|_{H^{\gamma}} \leqslant \frac{R}{3\left(1-r_{0}\right)} .
$$

Рассмотрим два вектора $\boldsymbol{q}^{1}, \boldsymbol{q}^{2} \in B_{\gamma}$ и найдем два решения $\omega^{1}, \omega^{2}$ (здесь $\omega^{i}=\left(v^{i}, p^{i}, \Theta^{i}, C^{i}\right.$ ), $i=1,2)$ системы $(55)-(57)$. Их разность $\omega^{1}-\omega^{2}$ удовлетворяет равенству

$$
\omega^{1}-\omega^{2}=A\left(\omega^{1}, \boldsymbol{q}^{1}\right)-A\left(\omega^{2}, \boldsymbol{q}^{1}\right)+A\left(\omega^{2}, \boldsymbol{q}^{1}\right)-A\left(\omega^{2}, \boldsymbol{q}^{2}\right),
$$

из которого следует

$$
\left\|\omega^{1}-\omega^{2}\right\|_{H^{\gamma}} \leqslant r_{0}\left\|\omega^{1}-\omega^{2}\right\|_{H^{\gamma}}+c_{1}\left\|\boldsymbol{q}^{1}-\boldsymbol{q}^{2}\right\|_{L_{q}(0, \gamma)}
$$

И

$$
\left\|\omega^{1}-\omega^{2}\right\|_{H^{\gamma}} \leqslant \frac{c_{1}}{1-r_{0}}\left\|\boldsymbol{q}^{1}-\boldsymbol{q}^{2}\right\|_{L_{q}(0, \gamma)} .
$$

Пусть $\gamma \leqslant \gamma_{1}$. Умножим уравнение (51) скалярно на вектор $\varphi_{k}$ и проинтегрируем результат по $G$, считая, что условия (54) выполнены. Как следствие получаем равенство

$$
\begin{gathered}
\tilde{\psi}_{k}^{\prime}-\int_{G}\left(\left(\sum_{i, j=1}^{n} a_{i j} C_{1 x_{i} x_{j}}+\sum_{j=1}^{n} a_{j} C_{1 x_{j}}+a_{0} C_{1}\right), \varphi_{k}(x)\right) d x= \\
=\left(g_{c}, \varphi_{k}\right)_{0}-\int_{G}\left(g_{c}+\sum_{j=1}^{n} c^{j} v_{j}, \varphi_{k}\right) d x+\sum_{j=1}^{s} q_{j} \int_{G}\left(f_{j}, \varphi_{k}\right) d x
\end{gathered}
$$

где

$$
\left(g_{c}, \varphi_{k}\right)_{0}=\int_{G}\left(g_{c}, \varphi_{k}\right) d x .
$$


Это равенство может быть переписано в виде

$$
\boldsymbol{q}=B^{-1} S_{0}(\boldsymbol{q})=S(\boldsymbol{q}),
$$

где $k$-я координата $s_{k}$ вектора $S_{0}$ записывается в виде

$$
\begin{gathered}
\alpha_{k}(\boldsymbol{q})=-\int_{G}\left(\left(\sum_{i, j=1}^{n} a_{i j} C_{1 x_{i} x_{j}}+\sum_{j=1}^{n} a_{j} C_{1 x_{j}}+a_{0} C_{1}\right), \varphi_{k}(x)\right) d x+\int_{G}\left(g_{c}+\sum_{j=1}^{n} c^{j} v_{j}, \varphi_{k}\right) d x, \\
s_{k}=F_{k}+\alpha_{k}(\boldsymbol{q}), \quad F_{k}=\tilde{\psi}_{k}^{\prime}-\left(g_{c}, \varphi_{k}\right)_{0} .
\end{gathered}
$$

Вектор-функции $C_{1}, v$, входящие в выражение $S(\boldsymbol{q})$, выражаются через вектор $\boldsymbol{q}$ и решение системы (37). Выберем параметр $R_{0}$ и соответственно параметр $R$. Положим $F=\left(F_{1}, F_{2}, \ldots, F_{n}\right)$ и определим $R_{0}=2\left\|B^{-1} F\right\|_{L_{q}(0, T)}$. Тогда параметр $R$, определенный выше, вычисляется через величину $R_{0}$ и нормы исходных данных. По параметру $R$ мы определили величину $\gamma_{1}$. Величина $\gamma_{1}$ будет зависеть только от норм данных. Тогда при всех $\gamma \leqslant \gamma_{1}$ оператор $S$ определен корректно.

Докажем разрешимость уравнения (43). Пусть $\boldsymbol{q}^{1}, \boldsymbol{q}^{2} \in B_{\gamma}$, где $\gamma \leqslant \gamma_{1}$. Приведем необходимые оценки и покажем, что оператор $S$ переводит шар $B_{\gamma}$ в себя и является в нем сжимающим, если параметр $\gamma$ мал. Основные слагаемые, входящие в функцию $\alpha_{k}$, имеют вид

$$
J_{k}=\int_{G}\left(a_{i j} C_{1 x_{i} x_{j}}, \varphi_{k}(x)\right) d x .
$$

Возьмем $\varepsilon<\min \left(1 / r, \varepsilon_{0}\right)$ (см. условие $\left.(19)\right)$. Пусть символ $\stackrel{\circ}{W_{r}^{s}}(G), s \leqslant 1$, обозначает пространство функций из $W_{r}^{s}(G)$, обращающихся в нуль на $\Gamma$ при $s>1 / r$, a $W_{p}^{-s}(G)$ - двойственное пространство. В силу двойственности (неравенство Щварца) имеем

$$
\left|J_{k}\right| \leqslant\left\|C_{1 x_{i} x_{j}}\right\|_{W_{q}^{-\varepsilon}(G)}\left\|a_{i j}^{*} \varphi_{k}\right\|_{W_{r}^{\varepsilon}(G)}, \quad \frac{1}{r}+\frac{1}{q}=1,
$$

где $a_{i j}^{*}$-сопряженная матрица. Ее элементы (см. условие (19)) принадлежат классу $C\left([0, T] ; C^{\varepsilon_{0}}(\bar{G})\right)$. Тогда в соответствии с теоремой о точечных мультипликаторах (см. [55, теорема 3.3.2, с. 198] (можно также использовать определение нормы) имеем

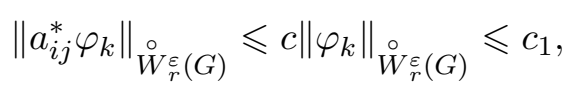

где $c, c_{1}$ - некоторые постоянные (не зависящие от времени). Отметим, что $\stackrel{\circ}{W} \underset{r}{\varepsilon}(G)=W_{r}^{s}(G)$ при $s<1 / r$, поэтому правая часть предыдущего неравенства конечна. С другой стороны, имеет место неравенство

$$
\left\|C_{1 x_{i} x_{j}}\right\|_{W_{q}^{-\varepsilon}(G)} \leqslant c\left\|C_{1}\right\|_{W_{q}^{2-\varepsilon}(G)} .
$$

Неравенства такого вида возникают часто. Они вытекают из интерполяционных свойств линейных операторов. Поясним доказательство. Рассмотрим отображение $M: v \rightarrow v_{x_{j}}$. Очевидно, $M \in L\left(L_{q}(G), W_{q}^{-1}(G)\right) \cap L\left(W_{q}^{1}(G), L_{q}(G)\right)$. Следовательно,

Однако

$$
M \in L\left(\left(L_{q}(G), W_{q}^{-1}(G)\right)_{\theta, q},\left(W_{q}^{1}(G), L_{q}(G)\right)_{\theta, q}\right), \quad \theta \in(0,1) .
$$

$$
\left(W_{q}^{1}(G), L_{q}(G)\right)_{\theta, q}=W_{q}^{1-\theta}(G), \quad\left(L_{q}(G), W_{q}^{-1}(G)\right)_{\theta, q}=W_{q}^{-\theta}(G)
$$

(см. [54]). Таким образом, имеет место неравенство

$$
\left\|v_{x_{j}}\right\|_{W_{q}^{-\varepsilon}(G)} \leqslant c\|v\|_{W_{q}^{1-\varepsilon}(G)} .
$$

Отсюда и вытекает неравенство (65). Из полученных неравенств вытекает оценка

$$
\left\|J_{k}\right\|_{L_{q}(0, \gamma)} \leqslant c\left\|C_{1}\right\|_{L_{q}\left(0, \gamma ; W_{q}^{2-\varepsilon}(G)\right)} .
$$

Далее используем интерполяционные неравенства (см. [54]) и лемму 1. Получаем

$$
\left\|J_{k}\right\|_{L_{q}(0, \gamma)} \leqslant c\left\|C_{1}\right\|_{L_{q}\left(0, \gamma ; W_{q}^{2-\varepsilon}(G)\right)} \leqslant c_{1}\left\|C_{1}\right\|_{L_{q}\left(Q^{\gamma}\right)}^{\varepsilon}\left\|C_{1}\right\|_{L_{q}\left(0, \gamma ; W_{q}^{2}(G)\right)}^{2-\varepsilon} \leqslant c_{2} \gamma^{\varepsilon}\left\|C_{1}\right\|_{W_{q}^{2,1}\left(Q^{\gamma}\right)} .
$$


Оставшиеся слагаемые в выражении $\alpha_{k}$ оцениваются гораздо проще. Рассмотрим, например, выражение

$$
I_{k}=\int_{G}\left(a_{j} C_{1 x_{j}}, \varphi_{k}(x)\right) d x .
$$

Имеем

$$
\left|I_{k}\right| \leqslant c\left\|\varphi_{k}(x)\right\|_{L_{r}(G)}\left\|a_{j} C_{1 x_{j}}\right\|_{L_{q}(G)} \leqslant c_{1}\left\|a_{j}\right\|_{L_{q}(G)}\left\|C_{1 x_{j}}\right\|_{L_{\infty}(Q)} .
$$

Далее, обращаясь к лемме 2 получим неравенство

$$
\left\|I_{k}\right\|_{L_{q}(0, \gamma)} \leqslant c_{2} \gamma^{\beta}\left\|C_{1}\right\|_{W_{q}^{2,1}\left(Q^{\gamma}\right)}
$$

где $\beta$ - некоторая положительная постоянная. Оставшиеся слагаемые в выражении $\alpha_{k}(\boldsymbol{q})$ оцениваются аналогично. Положим $\boldsymbol{\alpha}=\left(\alpha_{1}, \alpha_{2}, \ldots, \alpha_{s}\right)^{T}$. Таким образом, можем сказать, что найдется такое число $\beta_{0}>0$, что имеет место неравенство

$$
\left\|B^{-1} \boldsymbol{\alpha}(\boldsymbol{q})\right\|_{L_{q}(0, \gamma)} \leqslant c \gamma^{\beta_{0}}\left(\left\|C_{1}\right\|_{W_{q}^{2,1}\left(Q^{\gamma}\right)}+\|v\|_{W_{q}^{2,1}\left(Q^{\gamma}\right)}\right) \leqslant C(R) \gamma^{\beta_{0}} .
$$

Теперь возьмем два различных вектора $\boldsymbol{q}^{1}, \boldsymbol{q}^{2}$. Запишем разность $S_{0}\left(\boldsymbol{q}^{1}\right)-S_{0}\left(\boldsymbol{q}^{2}\right)$. Обозначим через $C^{i}, v^{i}$ (здесь $\left.v^{i}=\left(v_{1}^{i}, v_{2}^{i}, \ldots, v_{n}^{i}\right), i=1,2\right)$ решения системы (37), соответствующие этим векторам. Отметим, что данные векторы входят в функции $\alpha_{k}$ почти линейно. Поэтому легко видеть, что будет справедлива аналогичная оценка, но в правой части уже будут нормы разностей $C^{1}-C^{2}$, $v^{1}-v^{2}$. Используя (41), получим

$$
\left\|B^{-1} \boldsymbol{\alpha}\left(\boldsymbol{q}^{1}\right)-B^{-1} \boldsymbol{\alpha}\left(\boldsymbol{q}^{2}\right)\right\|_{L_{q}(0, \gamma)} \leqslant c(R) \gamma^{\beta_{1}}\left\|\boldsymbol{q}^{1}-\boldsymbol{q}^{2}\right\|_{L_{q}(0, \gamma)},
$$

где $\beta_{1}$ - некоторая положительная постоянная. Используя определение величины $R_{0}$, имеем неравенство

$$
\|S(\boldsymbol{q})\| \leqslant R_{0} / 2+C(R) \gamma^{\beta_{0}}, \quad\left\|S\left(\boldsymbol{q}^{1}\right)-S\left(\boldsymbol{q}^{2}\right)\right\|_{L_{q}(0, \gamma)} \leqslant c(R) \gamma^{\beta_{1}}\left\|\boldsymbol{q}^{1}-\boldsymbol{q}^{2}\right\|_{L_{q}(0, \gamma)} .
$$

Остается выбрать такую постоянную $\gamma_{2} \leqslant \gamma_{1}$, что если $\gamma \leqslant \gamma_{2}$, то

$$
C(R) \gamma^{\beta_{0}} \leqslant \frac{R_{0}}{2}, \quad c(R) \gamma^{\beta_{1}} \leqslant \frac{1}{2} .
$$

Тогда при $\gamma \leqslant \gamma_{2}$ уравнение (43) имеет единственное решение в шаре $B_{\gamma}$. Векторы $C_{1}, v$ и функцию $\Theta_{1}$ находим как решение системы (37). Покажем, что условия переопределения для функции $C_{1}$ выполняются. Умножим уравнение (31) скалярно на вектор-функцию $\varphi_{k}$ и проинтегрируем результат по $G$. Вычитая полученное равенство из $k$-го уравнения в (42), получим равенство

$$
\int_{G}\left(C_{1 t}, \varphi_{k}\right) d x=\tilde{\psi}_{k},
$$

а это как раз и есть условие переопределения. Таким образом, мы построили решение нашей задачи локально по времени, причем параметр $\gamma_{2}$ зависит только от норм коэффициентов уравнений в $Q$.

Покажем, что решение продолжимо на весь промежуток $[0, T]$. Построим функцию $\boldsymbol{q}_{1}=\boldsymbol{q}$ при $t \in\left[0, \gamma_{2}\right]$ и $\boldsymbol{q}_{1}=0$ при $t>\gamma_{2}$. Построим также четные продолжения $v^{1}, C^{1}, \Theta^{1}, p^{1}$ решений $v$, $C_{1}, \Theta_{1}, p$ относительно точки $t=\gamma_{2}$. Таким образом, $v^{1}=v$ при $t \in\left[0, \gamma_{2}\right]$ и $v^{1}(x, t)=v\left(x, 2 \gamma_{2}-t\right)$ при $t \in\left[\gamma_{2}, 2 \gamma_{2}\right]$. Сделаем в системе (55)-(57) замены

$$
\boldsymbol{q}=\boldsymbol{q}_{1}+\boldsymbol{q}_{2}, \quad v=v^{1}+v_{2}, \quad p=p^{1}+p_{2}, \quad \Theta_{1}=\Theta_{2}+\Theta^{1}, \quad C_{1}=C_{2}+C^{1} .
$$

Получим систему (55)-(57) относительно функций $v_{2}, p_{2}, \Theta_{2}, C_{2}$, удовлетворяющих однородным начальным и граничным данным, с новой правой частью, которая обращается в нуль на $\left[0, \gamma_{2}\right]$ и определена на $\left[0,2 \gamma_{2}\right]$. В силу единственности решения задачи на $\left[0, \gamma_{2}\right]$ получаем, что векторфункция $\left(v_{2}, p_{2}, \Theta_{2}, C_{2}\right)$ равна нулю на $\left[0, \gamma_{2}\right]$, равно как и функция $\boldsymbol{q}_{2}$. Используя этот факт, мы повторяем рассуждения уже на промежутке $\left[\gamma_{2}, 2 \gamma_{2}\right]$, причем без ограничения общности можем считать, что все оценки справедливы с теми же постоянными $c_{2}, \ldots, c_{6}$ (однако радиус шара, в котором ищется решение, изменится). Это дает возможность доказать существование решений 
системы $(55)-(57),(64)$ на промежутке $\left[0,2 \gamma_{2}\right]$. Далее, повторяя рассуждения, установим разрешимость системы на сегменте $\left[0,3 \gamma_{2}\right]$ и т. д. Таким путем устанавливается разрешимость задачи на всем сегменте $[0, T]$.

Замечание 1. Утверждения теорем 4, 5 остаются в силе, если вместо условий Дирихле мы будем рассматривать другие краевые условия для вектор-функции $u$ в уравнении (1), и имеет место соответствующий аналог теоремысоответствующий аналог теоремы 1.

\section{СПИСОК ЛИТЕРАТУРЫ}

1. Алексеев Г. В. Оптимизация в стационарных задачах тепломассопереноса и магнитной гидродинамики. - М.: Научный мир, 2010.

2. Алифанов О. М., Артюхин Е. А., Ненарокомов А. В. Обратные задачи в исследование сложного теплообмена. - М.: Янус-К, 2009.

3. Искендеров А. Д., Ахундов А. Я. Обратная задача для линейной системы параболических уравнений// Докл. РАН. - 2005. - 424, № 4. - С. 442-444.

4. Камынин В. Л., Франчини Э. Об одной обратной задаче для параблолического уравнения высокого порядка// Мат. заметки. - 1998. - 64, № 5. - С. 680-691.

5. Кожанов А. И. Параболические уравнения с неизвестным коэффициентом, зависящим от времени// Ж. вычисл. мат. мат. физ. - 2005. - 45, № 12. - С. 2168-2184.

6. Короткова Е. М., Пятков С. Г. О некоторых обратных задачах для линеаризованной системы тепломассопереноса// Мат. тр. - 2014. - 17, № 2. - С. 142-162.

7. Ладыженская О. А., Солонников В. А., Уральщева Н. Н. Линейные и квазилинейные уравнения параболического типа. - М.: Наука, 1967.

8. Лыков А. В., Михайлов Ю. А. Теория тепло- и массопереноса. - Ленинград: Госэнергоиздат, 1963.

9. Полежаев В. И., Бунэ А. В., Верезуб Н. А. и др. Математическое моделирование конвективного тепломассообмена на основе уравнений Навье-Стокса. - М.: Наука, 1987.

10. Прилепко А. И., Иванков А. Л., Соловъев В. В. Обратные задачи для уравнений переноса и параболических уравнений// в кн.: Единственность, устойчивость и методы решения некорректных задач математической физики. - Новосибирск: Вычислительный центр СО РАН, 1984. - С. 137-142.

11. Пятков С. Г., Сафонов Е. И. О некоторых классах линейных обратных задач для параболических систем уравнений// Науч. вед. БелГУ. - 2014. - 35, № 7 (183). - С. 61-75.

12. Пятков С. Г., Сафонов Е. И. О некоторых классах линейных обратных задач для параболических систем уравнений// Сиб. электрон. изв. - 2014. - 11. - С. 777-779.

13. Пятков С. Г. О некоторых обратных задачах для операторно-дифференциальных уравнений первого порядка// Сиб. мат. ж. - 2019. - 60, № 1. - С. 183-193.

14. Солонников В. А. О краевых задачах для линейных параболических систем дифференциальных уравнений общего вида// Тр. Мат. ин-та им. В. А. Стеклова АН СССР. - 1965. - 83. - С. 3-163.

15. Abels $H$. Reduced and generalized Stokes resolvent equations in asymptotically flat layers. Part I: Unique solvability// J. Math. Fluid Mech. - 2005. - 7, № 2. - C. 201--222.

16. Abels $H$. Reduced and generalized Stokes resolvent equations in asymptotically flat layers. Part II: $H^{\infty}$ calculus// J. Math. Fluid Mech. - 2005. - 7, № 2. - C. 223-260.

17. Abels $H$. Bounded imaginary powers and $H^{\infty}$-calculus of the Stokes operator in unbounded domains// в кн.: Nonlinear Elliptic and Parabolic Problems: A Special Tribute to the Work of Herbert Amann. Basel: Birkhäuser Verlag, 2005. - C. 1-15.

18. Amann H. Linear and Quasilinear Parabolic Problems. - Basel: Birkhäuser Verlag, 2005.

19. Amann H. Compact embeddings of vector-valued Sobolev and Besov spaces// Glas. Mat. - 2000. - 35 (55). - C. 161--177.

20. Bejan A. Convection heat transfer. - New York: Wiley, 2004.

21. Belov Ya. Ya. Inverse Problems for Parabolic Equations. - Utrecht: VSP, 2002.

22. Cannon J. R. A class of non-linear nonclassical parabolic equations// J. Differ. Equ. - 1989. - 79, № 2. - C. 266-288.

23. Capatina A., Stavre R. A control problem in biconvective flow// J. Math. Kyoto Univ. - 1997. - 37, № 4. - C. $585-595$. 
24. Loucks D. P., van Beek E. et al. Water Resources Systems: Planning and Management. - Paris-Delft: UNESKO Publishing, 2005.

25. Denk R., Hieber M., Prüss J. R-boundedness, Fourier multipliers, and problems of elliptic and parabolic type// Mem. Am. Math. Soc. - 2003. - 166. - C. 1-114.

26. Desch W., Hieber M., and Prüss J. $L_{p}$-Theory of the Stokes equation in a half space// J. Evol. Equ. 2001. - 1. - C. 115-142.

27. Denk R., Hieber M., and Prüss J. Optimal $L_{p}-L_{q}$-estimates for parabolic boundary-value problems with inhomogeneous data// Math. Z. - 2007. - 257, № 1. - C. 193-224.

28. Ewing R. E., Lin T. A class of parameter estimation techniques for fluid flow in porous media// Adv. Water Res. - 1991. - 14, № 2. - C. 89-97.

29. Farwig R., Myong-Hwan R. Resolvent estimates and maximal regularity in weighted $L_{q}$-spaces of the Stokes operator in an infinite cylinder// J. Math. Fluid Mech. - 2008. - 10, № 3. - C. 352-387.

30. Farwig R., Myong-Hwan R. The resolvent problem and $H^{\infty}$-calculus of the Stokes operator in unbounded cylinders with several exits to infinity// J. Evol. Equ. - 2007. - 7, № 3. - C. 497-528.

31. Frölich $A$. The Stokes operator in weighted $L_{q^{-}}$-spaces, II: Weighted resolvent estimates and maximal $L_{p^{-}}$ regularity// Math. Ann. - 2007. - 339, № 2. - C. 287-316.

32. Giga Y., Sohr H. On the Stokes operator in exterior domains// J. Fac. Sci. Univ. Tokyo. Sect. IA. Math. - 1989. - 36, № 2. - C. 103-130.

33. Giga Y., Sohr H. Abstract $L_{p}$-estimates for the Cauchy problem with applications to the Navier-Stokes equations in exterior domains // J. Funct. Anal. - 1991. - 102, № 1. - C. 72-94.

34. Grisvard P. Equations operationnelles dans les de Banach et preblemes aux limites dans des ouverts cylindriques // Ann. Scu. Norm. Super. Pisa. - 1967. - 21, № 3. - C. 308-347.

35. Hazanee A., Lesnic D., Ismailov M. I., Kerimov N. B. Inverse time-dependent source problems for the heat equation with nonlocal boundary conditions// Appl. Math. Comput. - 2019. - 346. - C. 800-815.

36. Hasanov A., Hasanoglu S. Comparative analysis of linear and nonlinear models for ion transport problem in chronoamperometry// J. Math. Chem. - 2008. - 44, № 3. - C. 731-742.

37. Hussein M. S., Lesnic D. Simultaneous determination of time-dependent coefficients and heat source// Int. J. Comput. Meth. Eng. Sci. Mech. - 2016. - 17, № 5-6. - C. 401-411.

38. Joseph D. D. Stability of Fluid Motions. - Berlin-Heidelberg-New York: Springer-Verlag, 1976.

39. Isakov $V$. Inverse problems for partial differential equations. - Berlin-Heidelberg-New York: SpringerVerlag, 2006.

40. Ismailov M. I., Kanca F. Inverse problem of finding the time-dependent coefficient of heat equation from integral overdetermination condition data// Inv. Probl. Sci. Eng. - 2012. — 20, № 4. - C. 436-476.

41. Ismailov M., Erkovan S. Inverse problem of finding the coefficient of the lowest term in two-dimensional heat equation with Ionkin-type boundary condition// Ж. вычисл. мат. мат. физ. - 2019. — 59, № 5. C. 859 .

42. Isakov $V$. Inverse problems for equations of parabolic type. - Berlin: Springer, 2003.

43. Jing Li, Youjun Xu An inverse coefficient problem with nonlinear parabolic equation// J. Appl. Math. Comput. - 2010. - 34, № 1-2. - C. 195-206.

44. Joseph D. D. Stability of fluid motions, II. - Berlin-Heidelberg-New York: Springer-Verlag, 1976.

45. Kabanikhin S. I. Inverse and Ill-Posed Problems. - Berlin-Boston: de Gruyter, 2012.

46. Kerimov N. B., Ismailov M. I. An inverse coefficient problem for the heat equation in the case of nonlocal boundary conditions// J. Math. Anal. Appl. - 2012. - 396, № 2. - C. 546-554.

47. Kozhanov A. I. Composite type equations and inverse problems. - Utrecht: VSP, 1999.

48. Levandowsky M., Childress W. S., Hunter S. H., Spiegel E. A. A mathematical model of pattern formation by swimming microorganisms// J. Protozoology. - 1975. - 22, № 2. - C. 296-306.

49. Ozisik M. N., Orlando H. A. B. Inverse Heat Transfer. - New York: Taylor \& Francis, 2000.

50. Prilepko A. I., Orlovsky D. G., Vasin I. A. Methods for Solving Inverse Problems in Mathematical Physics. - New York: Marcel Dekker, 1999.

51. Pyatkov S. G., Safonov E. I. Some inverse problems for convection-diffusion systems of equations// Becth. ЮУрГУ. Сер. мат. модел. программ. - 2014. - 7, № 4. - С. 36-50.

52. Pyatkov S. G., Safonov E. I. Solvability of some inverse problems for the nonstationary heat-and-masstransfer system// J. Math. Anal. Appl. - 2017. - 446, № 2. - C. 1449-1465. 
53. Solonnikov V. A. Estimates of solutions of the Stokes equations in Sobolev spaces with a mixed norm// J. Math. Sci. - 2004. - 123, № 6. - C. 4637-4653.

54. Triebel H. Interpolation Theory. Function Spaces. Differential Operators. - Berlin: VEB Deutscher Verlag der Wissenschaften, 1978.

55. Triebel H. Theory of Function Spaces. - Basel: Birkhäuser-Verlag, 1983.

\section{Пятков Сергей Григорьевич}

Югорский государственный университет, Ханты-Мансийск

E-mail: pyatkovsg@gmail.com, s_pyatkov@ugrasu.ru 